\title{
ZNF1 Encodes a Putative C2H2 Zinc-Finger Protein Essential for Appressorium Differentiation by the Rice Blast Fungus Magnaporthe oryzae
}

\author{
Xiaofeng Yue, ${ }^{1}$ Yawei Que, ${ }^{1}$ Lin Xu, ${ }^{1}$ Shuzhen Deng, ${ }^{1}$ Youliang Peng, ${ }^{2}$ Nicholas J. Talbot, ${ }^{3}$ and \\ Zhengyi Wang ${ }^{1}$ \\ ${ }^{1}$ State Key Laboratory for Rice Biology, Institute of Biotechnology, Zhejiang University, Hangzhou 310029, People's Republic of \\ China; ${ }^{2}$ State Key Laboratory of Agrobiotechnology and MOA Key Laboratory of Plant Pathology, China Agricultural University, \\ Beijing, People's Republic of China; ${ }^{3}$ School of Biosciences, University of Exeter, Geoffrey Pope Building, Exeter EX4 4QD, \\ United Kingdom
}

Submitted 5 September 2015. Accepted 27 September 2015.

\begin{abstract}
The rice blast fungus Magnaporthe oryzae forms specialized infection structures called appressoria which are essential for gaining entry to plant tissue. Here, we report the identification of a novel nonpathogenic T-DNA-tagged mutant XF696 of $M$. oryzae with a single insertion in the promoter of ZNF1, which encodes a putative transcription factor (TF). Targeted gene deletion mutants of $Z N F 1$ are nonpathogenic and unable to develop appressoria. However, $\Delta z$ nf1 mutants still respond to exogenous cyclic AMP on hydrophilic surfaces and can sense hydrophobic surfaces, initiating the differentiation of germ tubes. Interestingly, $\Delta z n f 1$ mutants also produce significantly more conidia compared with the isogenic wild-type strain. Quantitative reverse-transcription polymerase chain reaction analysis and green fluorescent protein fusion experiments revealed that expression of ZNF1 was highly induced during germination and appressorium development in $M$. oryzae and potentially regulated by the Pmk1 mitogen-activated protein kinase pathway. We observed that $\Delta z$ nf1 mutants are affected in mitosis and impaired in mobilization and degradation of lipid droplets and glycogen reserves during appressorium differentiation. Site-directed mutagenesis confirmed that three of the four $\mathrm{C} 2 \mathrm{H} 2$ zinc-finger domains are essential for the function of Znf1. Taken together, we conclude that a $\mathrm{C2H} 2$ zinc-finger TF encoded by ZNF1 is essential for appressorium development by the rice blast fungus.
\end{abstract}

Magnaporthe oryzae is the causal agent of rice blast, the most destructive disease of cultivated rice worldwide (Ou 1985; Wilson and Talbot 2009). The disease is initiated when threecelled conidia attach to a rice leaf via wind or rain splash dispersal, where they germinate rapidly and form specialized infection structures, called appressoria, at the tips of germ tubes. The appressorium attaches strongly to the rice leaf and generates enormous turgor (up to $8 \mathrm{MPa}$ ) by accumulating osmolytes such as glycerol. Turgor is used to allow a narrow penetration peg to mechanically rupture the plant cuticle (de Jong et al. 1997; Howard et al. 1991). After penetration, the

Corresponding author: Z. Wang; E-mail: zhywang@zju.edu.cn

*The $\boldsymbol{e}$-Xtra logo stands for "electronic extra" and indicates that nine supplementary figures and one supplementary table are published online.

(C) 2016 The American Phytopathological Society fungus differentiates into bulbous invasive hyphae. Eventually, the fungus spreads through rice leaf cells and produces visible necrotic disease lesions on the rice leaf surface after 3 to 5 days (Talbot 2003). At this stage, abundant conidia are formed from aerial conidiophores that emerge from the lesion to reinitiate the disease cycle. Under suitable epidemic conditions, the infection cycle may occur many times during the rice growing season, resulting in rapid disease spread and local epidemics. Understanding the molecular mechanisms involved in conidiation and appressorium formation are necessary to develop novel and durable strategies for disease control.

In the past two decades, considerable efforts have been made to understand the molecular mechanisms that regulate appressorium morphogenesis and penetration in $M$. oryzae. It is now clear that initiation of appressorium formation is controlled by the cyclic AMP-protein kinase A (cAMP-PKA) pathway, and appressorium maturation and invasive growth are regulated by the Pmk1 mitogen-activated protein kinase (MAPK) pathway, respectively (Choi and Dean 1997; Lee and Dean 1993; Mitchell and Dean 1995; Xu and Hamer 1996; Xu et al. 1997; Zhao et al. 2005). Recently, several transcription factors (TF) that function downstream from the two signaling pathways have been identified, such as Som1 (Yan et al. 2011), Cdtf1 (Yan et al. 2011), Mstu1 (Nishimura et al. 2009; Yan et al. 2011), Mst12 (Park et al. 2002), and Mcm1 (Zhou et al. 2011). A second MAPK pathway, involving the Mps1 MAPK, is required for appressorium-mediated penetration but is dispensable for initial appressorium development (Xu et al. 1998; Zhao et al. 2005). Recently, it has been demonstrated that both the MADS-box TF Mig1 and APSES TF Swi6 interact with Mps1 and function downstream of the Mps1 pathway (Mehrabi et al. 2008; Qi et al. 2012). However, the downstream regulators of these signaling pathways, which are involved in conidiation and appressorium development, have not been characterized in detail.

There are approximately 500 putative TF-encoding genes in the $M$. oryzae genome, and those with $\mathrm{C} 2 \mathrm{H} 2$ zinc fingers constitute one of dominant families (Park et al. 2013). Up to now, however, only a small proportion of $\mathrm{C} 2 \mathrm{H} 2$ zinc-finger TF genes have been functionally characterized in filamentous fungi. M. oryzae Cos1, Con7, and Crz1 belong to the C2H2-type zincfinger TF family. Deletion of COS1 resulted in a conidiophoreless phenotype and mutants failed to produce conidia (Zhou et al. 2009). CON7 gene disruption mutants produced two types of abnormal conidia and were completely nonpathogenic, due 
to defects in appressorium formation (Odenbach et al. 2007), while Crz1 is essential for pathogenicity (Choi et al. 2009; Zhang et al. 2009).

In this study, we report the identification of a novel, nonpathogenic T-DNA mutant XF696 of M. oryzae. Analysis of the corresponding mutation confirmed a single T-DNA insertion within the promoter of $Z N F 1$, which encodes a putative $\mathrm{C} 2 \mathrm{H} 2$ zinc-finger TF. Deletion of ZNF1 resulted in serious defects in appressorium formation and pathogenicity and an increase in asexual sporulation, while hyphal growth of the mutants was not affected. Transcriptional expression of ZNF1 was highly induced during appressorium development but suppressed in $\Delta p m k 1$ mutants. Additionally, site-directed mutagenesis indicated that three of the four $\mathrm{C} 2 \mathrm{H} 2$ zinc-finger domains in $\mathrm{Znf} 1$ were essential for its proper function, consistent with its role as a TF. When considered together, we conclude that the $\mathrm{C} 2 \mathrm{H} 2$ zinc-finger TF gene $Z N F 1$ is essential for appressorium development by the rice blast fungus $M$. oryzae.

\section{RESULTS}

Identification of the T-DNA-tagged gene ZNF1.

To identify pathogenicity-related genes of $M$. oryzae, we constructed a T-DNA insertional mutagenesis library. All transformants were screened for impairment in pathogenesis by inoculating barley leaves ('Golden Promise') with mycelial plugs cut from 10-day-old cultures. In the assays, five mutants (XF577, XF696, XF1379, XF1521 and XF1557) incapable of causing disease on barley were identified from 2,200 T-DNA insertional transformants (Fig. 1A and B). To identify T-DNA integration sites in the mutants, genomic DNA flanking the integrated T-DNAs was amplified using thermal asymmetrical interlaced polymerase chain reaction (TAIL-PCR), as described previously (Liu and Chen 2007). The third-round PCR products were sequenced and patterns of T-DNA integration into these mutants were determined. Bioinformatics analysis showed that T-DNA insertion sites in XF577, XF1379, and XF1521 were
A

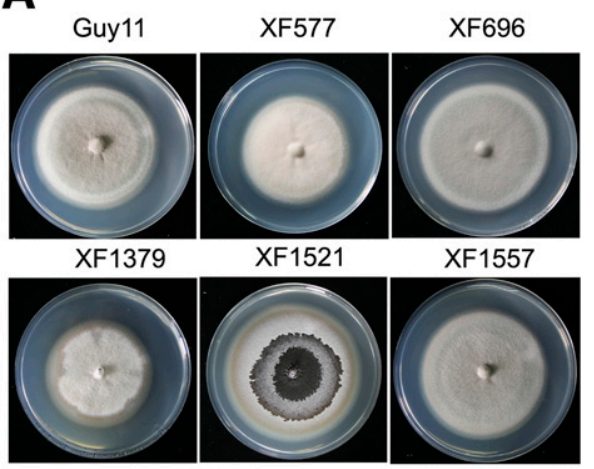

C



$\mathbf{E}$



B

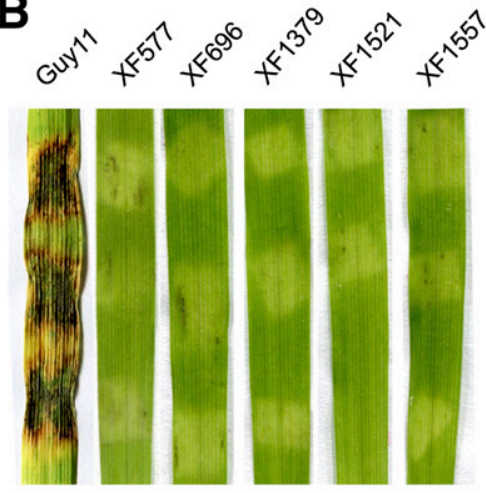

D

Genomic DNA (supercontig 12)



$\mathbf{F}$

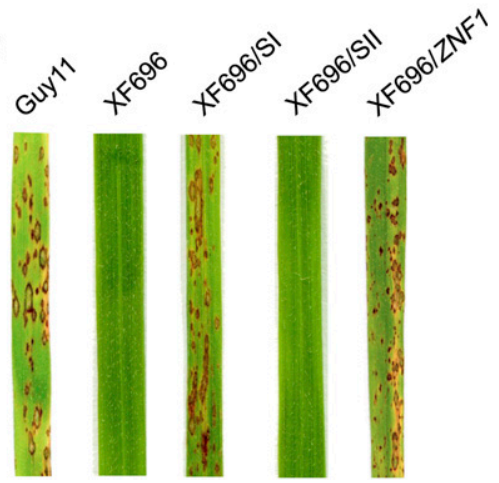

Fig. 1. Identification of T-DNA targeted gene ZNF1. A, Colonies of the wild-type Guy 11 and five T-DNA insertional mutants. B, Barley leaf segments were inoculated with mycelial plugs from different strains: Guy11, XF577, XF696, XF1379, XF1521, and XF-1557. C, Thermal asymmetrical interlaced polymerase chain reaction assays of the T-DNA insertion position in XF696 mutant. Lanes 1 to 3 present the third-round amplifications of genomic DNA flanking site of the integration T-DNA in the mutant and lane M = DNA marker. D, Position of the T-DNA insertion in XF696 and the structure of the locus of the predicted gene MGG_14931 (Broad Institute). An arrow indicates the T-DNA insertion position. Thick boxes and the thin line regions represent the predicted exons and introns, respectively. E, Two spliced variants (S I and S II) of ZNF1. S I was identical to the gene (XP_003719941) from the National Center for Biotechnology Information database. S II had a 127-bp DNA fragment missing at the C terminus of ZNF1. F, Reintroduction of either the S I isoform or fulllength open reading frame of ZNF1 into XF696 was able to restore the pathogenic defect of the mutant but S II could not. 
$482,1,733$, and 712 bp upstream of the translational start sites of BUF1 (MGG_02252), STU1 (MGG_00692), and MKK2 (MGG_06482), respectively, while the insertion site in XF1557 was 195 bp downstream of the translational start site of MST12 (MGG_12958). Because four of the T-DNA tagged genes have been functionally characterized previously (Chumley and Valent 1990; Nishimura et al. 2009; Park et al. 2002; Zhao et al. 2005), they were not selected for further investigation.

For the mutant XF696, the third-round TAIL-PCR amplification for the right border and the T-DNA integration pattern are shown in Figure 1C and D, respectively. The T-DNA insertion in the mutant was found at position $1436850+$, which is $158 \mathrm{bp}$ upstream of the translational start site, in a hypothetical gene (MGG_14931.6, Broad Ins.) located on supercontig 12 of chromosome VI. The $M G G \_14931$ locus is predicted to encode zinc-finger protein 32, with four consecutive $\mathrm{C} 2 \mathrm{H} 2$ zinc-finger domains at its $\mathrm{N}$ terminus. We named the T-DNA-tagged gene ZNF1 (for M. oryzae zinc-finger protein).

Znf1 showed 62.08, 55.43, 52.52, and 29.19\% amino acid identity with homologs from Colletotrichum graminicola GLRG_05053, Fusarium oxysporum FOIG_00328, Sclerotinia sclerotiorum SS1G_06088, and Neurospora crassa NCU03421, respectively. Each of the proteins has a highly conserved amino acid sequence in its $\mathrm{N}$ terminus, which contains four zinc-finger domains. However, Znf1 showed only $13.10 \%$ amino acid identity with human Znf32 (Gene ID: 7580) and no significant homologs were found in the Saccharomyces cerevisiae genome. Phylogenetic analysis of putative homologs of Znf1 from different filamentous fungal species is shown in Supplementary Figure S1

\section{ZNF1 transcripts have two alternatively spliced isoforms.}

To confirm the position and size of introns in ZNF1, a corresponding cDNA was obtained by reverse-transcription (RT)-PCR with primers C696F and C696R (Supplementary Table S1) and sequenced. Comparison of the cDNA and sequenced genomic DNA confirmed that ZNF1 possesses two different spliced isoforms or variants, which were named S I and S II. The $\mathrm{S} I$ isoform had an open reading frame (ORF) of 1,649 bp interrupted by three introns (240, 62, and $57 \mathrm{bp}$ ) and putatively encodes a 429 -amino-acid (aa) protein, which was $100 \%$ identical to the submission in the National Center for Biotechnology Information database (accession number XP_003719941) (Fig. 1E). Surprisingly, in the S II isoform, an additional spliced fragment ( $127 \mathrm{bp}$ ) without typical intron structures was found at the $\mathrm{C}$ terminus of the gene compared with S I (Fig. 1E). In the $M$. oryzae genome, the predicted gene $\left(M G G_{-} 14931.6\right)$ has an ORF of $1,651 \mathrm{bp}$ interrupted by three introns $(240,70$, and $279 \mathrm{bp}$ ) and putatively encodes a 353 -aa protein (Fig. 1D). However, the predicted splicing pattern of the gene as annotated in the genome database was not confirmed by our analysis. The detailed nucleotide differences among the predicted $M G G \_14931$, S I, and S II are shown in Supplementary Fig. S2.

To further understand the proportion of the two distinct ZNF1 isoforms, cDNA was amplified with primers C696F and C696R from the RT products of RNA extracted from vegetative mycelium, conidia, germinated conidia ( $4 \mathrm{~h}$ of incubation), and appressoria $(24 \mathrm{~h})$. In total, $67 \mathrm{cDNA}$ clones were isolated and sequenced. Interestingly, we found that both S I and S II isoforms were present in all fungal tissues tested. The frequency of the S I and S II variants was 73.13 and $26.87 \%$, respectively, indicating $\mathrm{S} I$ as the predominant spliced isoform in M. oryzae.

To determine the function of the two splicing isoforms, complementation vectors were constructed by ligating each cDNA (S I and S II) into pCB1532 under the native promoter (1.6 kb upstream DNA sequence of ZNF1). In addition, another complementation vector of $Z N F 1$ was constructed by amplifying a 3.22-kb genomic DNA fragment including a $1.65-\mathrm{kb}$ full-length ZNF1 ORF and its 1.6-kb upstream region using primers HB-F and HB-R and then cloning this into PCB1532. Complementation vectors were transformed into the T-DNA insertional mutant (XF696). Pathogenicity tests were carried out by spray inoculating rice ('CO-39') leaves with different transformants and the wild-type strain at a concentration of $5 \times 10^{4}$ conidia $\mathrm{ml}^{-1}$. These assays showed that either the full-length ZNF1 gene sequence or S I cDNA was able to complement the defects of the XF696 mutant and produce typical rice blast lesions (Fig. 1F). However, strains transformed with the S II cDNA remained nonpathogenic (Fig. 1F). therefore, these results suggest that the nonpathogenic phenotype of XF696 mutant was caused by the T-DNA integration and that S I but not the S II spliced variant of ZNF1 is necessary for the biological function of the gene in $M$. oryzae.

Yeast strains cotransformed with bait vector pGBKT7-Znf1 (S I) or (S II) and prey plasmid pGADT7 were able to grow on SD-Trp-Leu-His-Ade medium (Supplementary Fig. S3), suggesting that both Znf1 S I and S II isoforms have self- or autotranscriptional activation activity in yeast.

\section{The $\Delta z n f 1$ mutants are nonpathogenic.}

To further verify the function of $Z N F 1$, we performed a targeted gene deletion of ZNF1 using the gene replacement vector pKO-696 (Supplementary Fig. S4A). From 120 hygromycinresistant transformants obtained from several independent transformation experiments, eight $\Delta z n f 1$ mutants were identified by PCR amplification using primers Yq696F and Yq696R. Among them, two $\Delta z n f 1$ mutants, $\Delta z n f 1-89$ and -92, were selected and gene deletion events were confirmed by Southern blot analysis (Supplementary Fig. S4B) and RT-PCR amplification using primers C696-F and C696-R (data not shown). We then harvested conidia from $\Delta z n f 1$ mutants grown in 10-day complete medium (CM) cultures. Conidial suspensions were used to inoculate susceptible barley and rice leaves using either cut-leaf assays $\left(1 \times 10^{5}\right.$ conidia $\left.\mathrm{ml}^{-1}\right)$ or spray-inoculation assays $(5 \times$ $10^{4}$ conidia $\mathrm{ml}^{-1}$ ). In the cut-barley-leaf assays, the wild-type strain Guy11 and an ectopic transformant (ECT) caused severe infection on both intact and abraded barley (Fig. 2A). However, consistent with analysis of XF696, the $\Delta z n f 1-89$ and -92 mutants were nonpathogenic on susceptible barley seedlings, even when leaf surfaces were abraded to remove the surface cuticle (Fig. $2 \mathrm{~A}$ ) or wounded by a needle (data not shown), indicating that penetration and subsequent infectious growth of mutants was blocked by disruption of the ZNF1 gene. Similar results were observed in cut-rice-leaf assays (data not shown). Consistently, when 7-day-old barley or 2-week-old rice seedlings were spray inoculated with conidial suspensions of the strains, the wild-type strain and ECT caused typical rice blast lesions, whereas the $\Delta z n f 1$ mutants were completely nonpathogenic (Fig. 2B). We also performed rice root infection assays (Sesma and Osbourn 2004) and found that the $\Delta z n f 1$ mutants were unable to cause root disease (Fig. 2C). These data demonstrate that ZNF1 is essential for infectious growth and pathogenicity by $M$. oryzae in different tissue types.

To further ensure that the nonpathogenic phenotype observed in the $\Delta z n f 1$ mutants was associated with the gene replacement event, the full-length $Z N F 1$ ORF and its native promoter were reintroduced into the $\Delta z n f 1-89$ mutant. We obtained 20 complemented transformants from several fungal transformation experiments. All the transformants exhibited full virulence to barley and rice, although only the results of one complemented strain $\Delta z n f 1-89 \mathrm{C}$ are shown in Figure 2 . The other phenotypes of the $\Delta z n f l$ mutants, including conidiation and appressorium formation, were also fully complemented by reintroduction of the ZNF1 gene. 


\section{Deletion of ZNF1 increases conidiation in M. oryzae.}

Deletion of ZNF1 did not result in obvious phenotypic alterations in vegetative growth and colony pigment production in M. oryzae (Fig. 3A). Similar growth to the wild-type strain Guy 11 was also observed when $\Delta z n f 1$ mutants were grown on other media, such as potato dextrose agar, minimal medium, and liquid CM (data not shown). In addition, the $\Delta z n f 1$ mutants exhibited vegetative growth patterns similar to those of Guy 11 when grown on CM supplemented with different agents to induce external stresses, including osmotic stress (1.0 M sorbitol, $0.7 \mathrm{M} \mathrm{NaCl}$, or $1.0 \mathrm{M} \mathrm{KCl})$, oxidative stress $\left(5 \mathrm{mM} \mathrm{H}_{2} \mathrm{O}_{2}\right)$, and cell wall damage (Congo red at $200 \mathrm{\mu g} \mathrm{ml}^{-1}$ and $0.01 \%$ sodium dodecyl sulfate) (data not shown). These results suggest that Znf1 is not required for vegetative growth and pigment production.

In contrast, deletion of the ZNF1 gene caused a significant increase in conidiation in M. oryzae (Fig. 3B and C). Microscopy revealed denser aerial conidiophores formed by $\Delta z n f 1$ mutants compared with Guy 11 and the complemented strain $\Delta z n f 1-89 \mathrm{C}$ (Fig. 3B) . The $\Delta z n f 1$ mutants were grown on $\mathrm{CM}$ plates at $25^{\circ} \mathrm{C}$ for 10 days and conidia were carefully harvested. Consistently, the $\Delta z n f I-89$ and -92 mutants produced 1.21 and $1.29 \times 10^{7}$ conidia/plate, respectively, which was significantly more than $0.68 \times 10^{7}$ conidia/plate by the wild-type strain and $0.67 \times 10^{7}$ conidia/plate by $\Delta z n f 1-89 \mathrm{C}$ (Fig. $3 \mathrm{C}$ ). These data suggest that ZNF1 plays an important role in negative regulation of asexual sporulation in M. oryzae.

In M. oryzae, several TF-encoding genes, including COS1, HTF1 (HOX2), and CON7, have been shown to be involved in regulation of conidiophore formation or conidial production (Kim et al. 2009; Liu et al. 2010; Odenbach et al. 2007; Zhou et al. 2009). To investigate the effect of deletion of ZNF1 on expression of these genes, we carried out quantitative (q) RT-PCR analysis. The expression of COS1 was 2.66 -fold upregulated by the deletion of $Z N F 1$, whereas the expression level of $H T F 1$ and $C O N 7$ were not significantly affected (Fig. 3D).

To determine the role of ZNF1 in sexual reproduction, the wild-type Guy11 (MAT1-2) and $\triangle z$ zfl mutants were crossed with a standard tester strain, TH3 (MAT1-1), of M. oryzae to allow perithecium production. We observed numerous perithecia at the junctions of both the crosses of Guy $11 \times \mathrm{TH} 3$ and $\Delta z n f 1-89 \times$ TH3 (Fig. 3E), indicating that Znf1 is not required for fertility and development of fruiting bodies by M. oryzae.

A

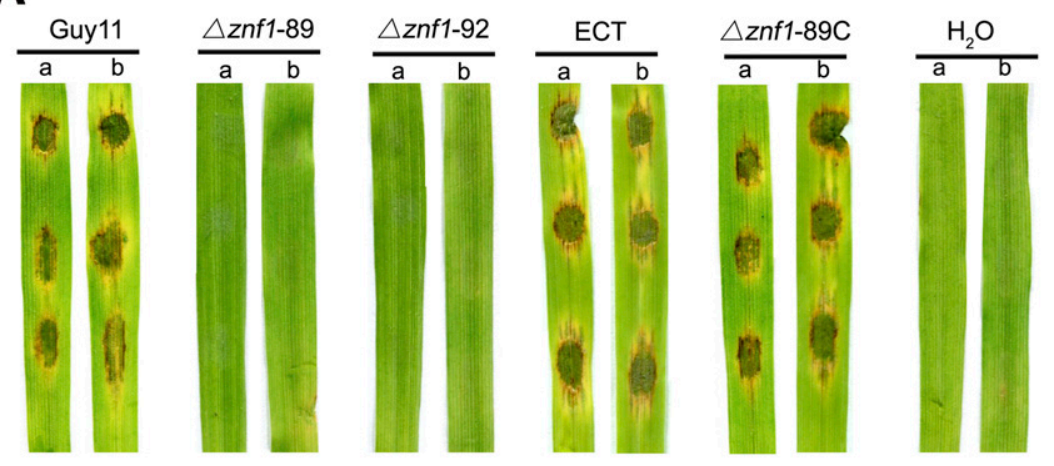

B
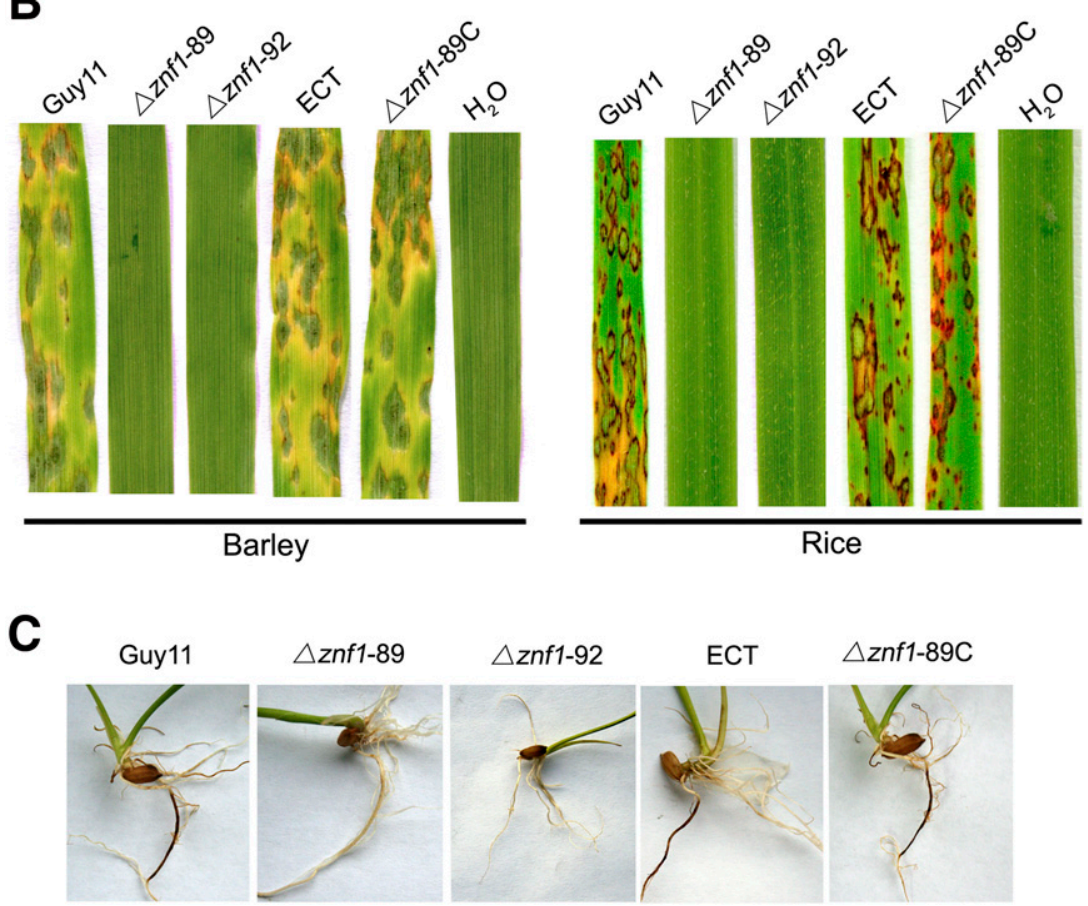

Fig. 2. The $\Delta z n f l$ mutants are unable to cause rice blast disease. A, Barley cut-leaf assays. With $30 \mu \mathrm{l}$ per drop, conidial suspension $\left(1 \times 10^{5} \mathrm{conidia}^{\mathrm{ml}} \mathrm{l}^{-1}\right)$ of each strain was inoculated on the 7-day-old intact leaves (a) and abraded leaves (b). ECT = ectopic transformant. B, Spray-inoculation assays. Seedlings of barley and rice were spray inoculated with $8 \mathrm{ml}$ of conidial suspension $\left(5 \times 10^{4}\right.$ conidia ml $\left.{ }^{-1}\right)$ of each strain. C, Root infection assays. Mycelial plugs were placed on rice roots and incubated for 7 days at $28^{\circ} \mathrm{C}$. 
We conclude that Znf1 plays an important role in negative regulation of asexual sporulation but not in vegetative growth and sexual development by $M$. oryzae.

\section{The $\Delta z$ nf1 mutants are unable to form appressoria.}

To determine the roles of ZNF1 in appressorium development and plant cuticle penetration, conidial suspensions were incubated on hydrophobic surfaces to induce appressorium formation, and on onion and barley epidermis to observe cuticle penetration. Conidia were harvested from 10-day-old CM cultures and appressorium formation investigated by incubating conidial suspension on hydrophobic GelBond. After $2 \mathrm{~h}$ of incubation, more than $90 \%$ of the conidia of all tested strains, including Guy11, $\Delta z n f 1-89 \mathrm{C}$, and $\Delta z n f 1$ mutants, were able to germinate to form germ tubes and then start hooking, indicating that deletion of ZNF1 did not affect conidial germination and initial germ tube differentiation. By $12 \mathrm{~h}$ postinoculation, numerous melanized appressoria were formed from short germ tubes of Guy11 and the complemented strain, whereas the $\Delta z n f 1-89$ mutant could only form subapical swollen structures (Fig. 4A). By $24 \mathrm{~h}$ or even $48 \mathrm{~h}$ postinoculation, no typical appressoria of the mutant were observed. Consistently, the wildtype and the complemented $M$. oryzae strains produced normal melanized appressoria on onion or barley epidermis surfaces and formed invasive hyphae in plant epidermal cells (Fig. 4B). By contrast, the $\Delta z n f 1-89$ mutant failed to form appressoria or undergo invasive hyphae, although they could still sense hydrophobic surfaces and undergo hooking (Fig. 4B). We conclude that ZNF1 is essential for appressorium development, maturation, and cuticle penetration by $M$. oryzae.

Recently, it has been shown that $M$. oryzae can form appressorium-like structures at hyphal tips on hydrophobic

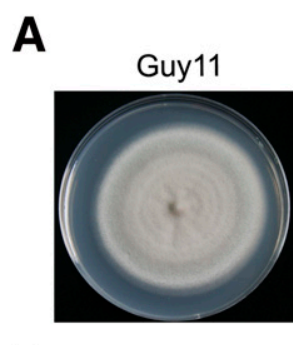

B


$\triangle z n f 1-89$

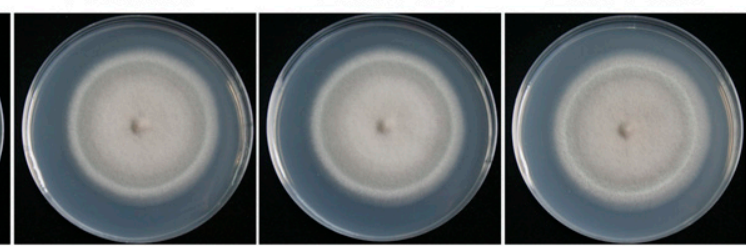

C



D

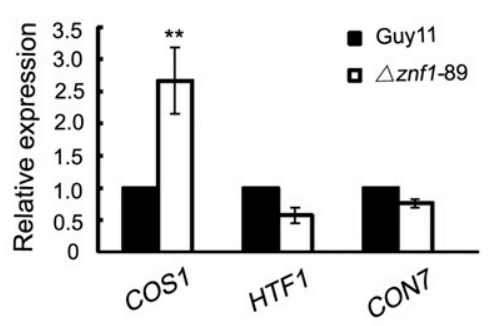

E



Fig. 3. ZNF1 negatively regulates asexual sporulation in Magnaporthe oryzae. A, Colonies of the wild-type strain Guy11, ZNF1 deletion mutants ( $\triangle$ znf1-89 and -92), and complemented strain $(\Delta z n f 1-89 \mathrm{C})$. Photographs were taken after incubating on complete medium $(\mathrm{CM})$ at $25^{\circ} \mathrm{C}$ for 10 days. All of the strains performed similar patterns of vegetative growth and pigment production. B, Microscopic observation of conidial development. The $\Delta z$ nf $1-89$ and -92 mutants formed more conidiophores and conidia than Guy11 and $\Delta z n f 1-89 \mathrm{C}$. All the strains grown on straw and corn medium for 7 days were examined by light microscopy. Scale bar $=50 \mu \mathrm{m}$. C, Statistical analysis of conidial production. The mutants ( $\Delta z n f 1-89$ and -92$)$ produce significantly more conidia than Guy 11 and $\triangle z n f 1-89 \mathrm{C}$ on $\mathrm{CM}$ at $25^{\circ} \mathrm{C}$ for 10 days. Error bars represent standard deviation. D, Quantitative reverse-transcription polymerase chain reaction analysis of transcriptional expression of COS1, HTF1/HOX2, and CON7 genes in the $\triangle z$ nf1-89 mutant. The relative expression level of COS1 was significantly increased in the $\Delta z n f 1-89$ mutant compared with Guy11. RNA of each strain was extracted from mycelium grown in liquid CM for 2 days. E, Fertility assay. Both of the crosses Guy $11 \times \mathrm{TH} 3$ and $\Delta z n f 1-89 \times \mathrm{TH} 3$ formed numerous perithecia on oatmeal medium after 1 month of incubation at $19^{\circ} \mathrm{C}$ in light. Asterisks $(\mathrm{C}$ and $\mathrm{D})$ indicate a significant difference $(* *, P<0.01)$ 
surfaces (Kong et al. 2013). We prepared hyphal suspensions to induce formation of appressorium-like structures on hydrophobic surfaces. Similarly, although swollen structures were observed, as found on conidial germ tubes, hyphal tips were unable to form typical appressorium-like structures by the $\Delta z n f 1 \mathrm{mu}-$ tants, whereas the wild-type strain and the complemented strain formed melanized appressorium-like structures (Fig. 4A).

\section{The $\Delta z$ nf1 mutants can respond to exogenous cAMP or IBMX but fail to form mature appressoria.}

M. oryzae is unable to form appressoria on hydrophilic surfaces. Addition of exogenous cAMP or IBMX (a phosphodiesterase inhibitor) to germinating conidia of the blast fungus can, however, induce appressorium formation on such noninductive surfaces (Lee and Dean 1993). To understand the effect of cAMP on appressorium formation in $\Delta z n f l$ mutants, $5 \mathrm{mM}$ exogenous cAMP or $2.5 \mathrm{mM}$ IBMX was added to conidial suspensions to induce appressorium formation on hydrophilic surfaces. In the absence of cAMP or IBMX, conidia of both the wild-type strain and the $\Delta z n f 1-89$ mutant produced undifferentiated hyphae on hydrophilic surfaces after $24 \mathrm{~h}$ of incubation (Fig. 5A). Upon addition of exogenous cAMP or IBMX, conidia of the wild-type strain formed melanized appressoria. Interestingly, exogenous cAMP or IBMX could induce hooking or swollen germ tube tip structures in $\Delta z n f 1$ mutants (Fig. 5A). However, these structures were unable to form mature appressoria, even after extended incubation or higher concentrations of cAMP (data not shown). On hydrophobic surfaces, adding exogenous cAMP or IBMX to conidial suspension of the $\Delta z n f 1-89$ mutant also elevated the frequency of germ tube swelling (Fig. 5B). Interestingly, new germ tubes were frequently generated from the swollen structures (Fig. 5B). These results suggest that $\Delta z n f 1$ mutants can respond to a cAMP signal but fail to complete formation of mature appressoria.

\section{Expression of $Z N F 1$ is highly induced during appressorium maturation.}

Because ZNF1 is necessary for appressorium formation and maturation in $M$. oryzae (Figs. 4 and 5), we conducted qRTPCR analysis to investigate differential expression of ZNF1 during appressorium development. Vegetative mycelium was harvested from 2-day-old CM liquid culture of the wild-type strain Guy11. Conidia were collected from 10-day-old CM cultures and allowed to form appressoria on hydrophobic surfaces, and appressoria were harvested at different time intervals: $4,8,16$, and $24 \mathrm{~h}$. qRT-PCR analysis showed that expression of ZNF1 was significantly induced during appressorium development (Fig. 6A).

To further investigate temporal expression of $Z N F 1$, a promoter fusion to green fluorescent protein (GFP) using $1.6 \mathrm{~kb}$ of the native promoter of $Z N F 1$ and the construct subsequently transformed into the $\Delta z n f 1-89$ mutant. Transformants expressing GFP were selected and fluorescence was examined under a confocal laser-scanning microscope. GFP fluorescence was
A
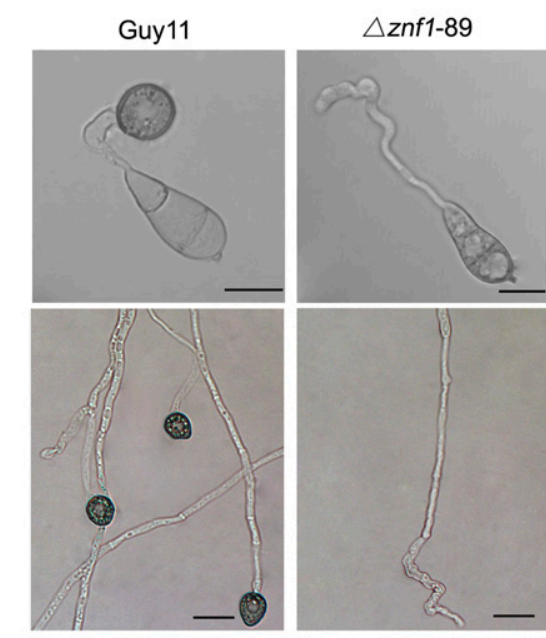

B

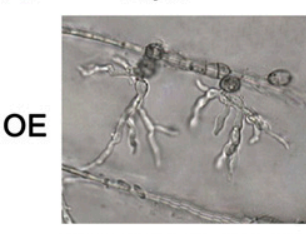

BE



$\triangle$ znf1-92
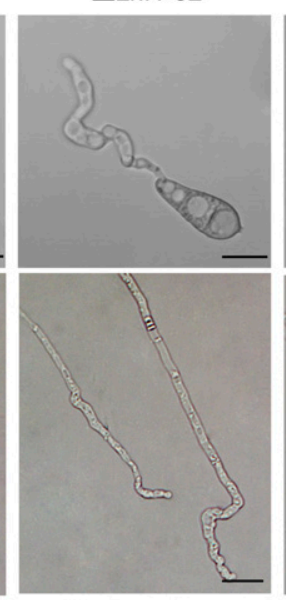

$\triangle$ znf1-92
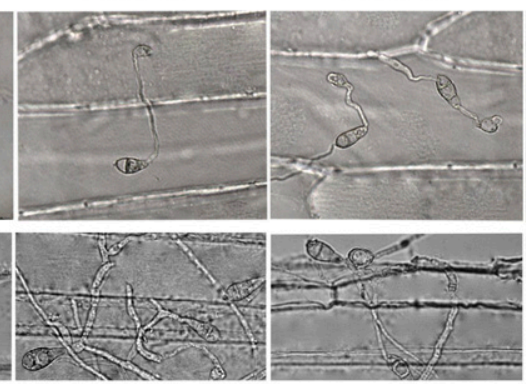

$\triangle$ znf1-89C
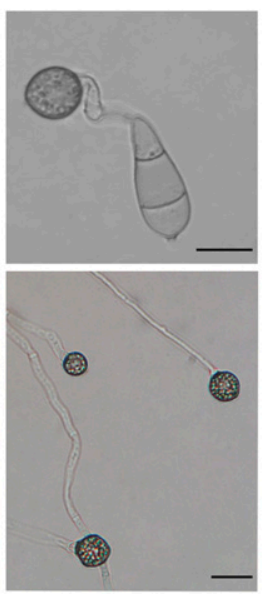

$\triangle$ znf1-89C
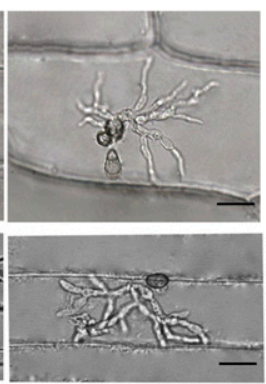

Fig. 4. ZNF1 is essential for appressorium development in Magnaporthe oryzae. A, Microscopic observation of appressorium development by germ tubes (top panel) and hyphal tips (bottom panel). The wild-type strain (Guy11) and the complemented strain $(\Delta z n f 1-89 \mathrm{C})$ were able to produce melanized appressoria on germ tubes and appressorium-like structures on hyphal tips, whereas the mutants $\Delta z n f 1-89$ and -92, only formed swollen structures. Appressorium formation was examined $12 \mathrm{~h}$ after incubation of the conidial suspension on hydrophobic GelBond. Scale bars of top panel $=10 \mu \mathrm{m}$ and of bottom panel $=50 \mu \mathrm{m}$. $\mathbf{B}$, Penetration assay on onion epidermal (OE) and barley epidermal (BE) cells. The wild-type strain and $\Delta z n f 1-89 \mathrm{C}$ were able to penetrate the epidermal cells and produced abundant invasive hyphae. The two mutants ( $\Delta z n f 1-89$ and -92) failed to form appressoria and no penetration events were observed. Photographs were taken after $24 \mathrm{~h}$ of inoculation on onion epidermis and $36 \mathrm{~h}$ on barley. Scale bars $=20 \mu \mathrm{m}$. 
observed mostly in cytoplasm of mycelium, conidia, germ tubes, and appressoria. Consistent with the results of qRT-PCR, brighter GFP fluorescence was observed during conidial germination (Supplementary Fig. S5). These results suggest that ZNF1 is highly expressed during conidial germination and appressorium maturation in $M$. oryzae.

\section{Expression of ZNF1 is significantly inhibited} during appressorium formation in $\Delta p m k 1$ mutants.

The $\Delta z n f l$ mutants exhibited similar phenotypes to $\Delta p m k 1$ mutants (Xu and Hamer 1996), such as defects in appressorium formation, penetration, and infectious growth. We reasoned that ZNF1 might be regulated by the Pmk1-MAPK pathway and, therefore, expression of ZNF1 may be affected by deletion of $P M K 1$ in $M$. oryzae. To test this idea, we performed qRT-PCR analysis to measure expression of ZNF1 in $\Delta p m k 1$ mutants. Conidial suspension of a $\Delta p m k 1$ mutant was placed on hydrophobic GelBond surfaces, and germinated conidia and germ tubes were harvested at $4,8,16$, and $24 \mathrm{~h}$ postincubation. The results showed that the expression level of ZNF1 in mycelium of the $\Delta p m k 1$ mutant was not significantly altered compared with the wild-type strain Guy11 (Fig. 6B). However, we found that expression of ZNF1 in the $\triangle p m k 1$ mutant was significantly downregulated during conidial germination and appressorium development compared with that in the wild-type strain (Fig. $6 \mathrm{~B})$, suggesting that $Z N F 1$ is regulated by the MAPK-Pmk1 pathway in M. oryzae. Additionally, the expression of the ZNF1 gene in several other mutants, including $\triangle m a g B, \Delta m a c 1, \Delta c p k A$, and $\Delta$ soml (key components in cAMP-PKA pathway), was also downregulated compared with the wild-type strain (Supplementary Fig. S6). These results are consistent with expression of ZNF1 being affected by the Pmk1-MAPK and cAMP-PKA pathways.

In light of the potential interaction between $\mathrm{Znf} 1$ and the Pmk1 MAPK, we carried out yeast two-hybrid (Y2H) experiments to determine whether a possible physical interaction occurs between $\mathrm{Znf1}$ and Pmk1 in $M$. oryzae. The results showed that Znf1 cannot directly interact with Pmk1 in $\mathrm{Y} 2 \mathrm{H}$ assays (data not shown).

\section{The $\Delta z n f 1$ mutants are impaired in nuclear degradation during appressorium formation.}

It has been previously reported that mitosis is affected during appressorium formation in the $\Delta p m k 1$ mutants (Veneault-Fourrey et al. 2006; Xu and Hamer 1996). To determine whether deletion of ZNF1 gene results in similar effects on mitosis, the histone $\mathrm{H} 1$ red fluorescent protein (RFP) fusion vector was introduced into the $\Delta z n f 1-89$ mutant. Conidia from a resulting transformant ( $\Delta z n f 1-89 \mathrm{R})$ expressing RFP were placed on hydrophobic GelBond surfaces and fluorescence was examined under a confocal laser-scanning microscope. By $24 \mathrm{~h}$ after incubation, we observed only a single nucleus in an appressorium of the wild-type strain but numerous nuclei in the conidium and germ tube of the $\Delta z n f 1-89 \mathrm{R}$ mutants, indicating that, as in $\Delta p m k 1$ mutants, cell cycle progression and nuclear degradation are severely affected in $\Delta z n f l$ mutants (Fig. 7). In addition, supplementation with $5 \mathrm{mM}$ exogenous cAMP partially restored the ability to break down nuclei (Fig. 7).


Fig. 5. The $\Delta z n f 1$ mutants can respond to exogenous cAMP or IBMX but fail to form mature appressoria. Conidia of the wild-type strain Guy 11 and the $\Delta z n f 1-$ 89 mutant were allowed to germinate on $\mathbf{A}$, hydrophilic and $\mathbf{B}$, hydrophobic GelBond film surfaces for $24 \mathrm{~h}$ in darkness. By adding exogenous $5 \mathrm{mM}$ cAMP and $2.5 \mathrm{mM}$ IBMX, the $\Delta z n f 1-89$ mutant was able to form hooking or swelling globular structures at the tips of germ tubes on hydrophilic surfaces (A) or elevated the level of germ tube swelling and melanization on hydrophobic surfaces (B). Scale bar $=20 \mu \mathrm{m}(\mathrm{A}$ and B). 
Nuclear degradation during appressorium formation requires autophagic cell death of the conidium (Veneault-Fourrey et al. 2006). To investigate whether autophagy was affected by loss of ZNF1, a GFP-Atg8 fusion vector was transformed into the $\Delta z n f 1-89$ mutant. We observed that autophagy in conidia of the resulting transformants was affected during a time course of appressorium development, largely as a consequence of the conidium failing to collapse during germ tube extension of the $\Delta z n f 1-89$ mutant. Autophagosomes continued to increase in abundance in spores and in elongated germ tubes of the fungus, in contrast to Guy11, which underwent autophagic cell death of conidia, followed by an autophagic burst in the developing appressorium, as previously reported (Kershaw and Talbot 2009) and as shown in Supplementary Figure S7.

\section{Znf1 is involved in mobilization and metabolism of lipids and glycogen reserves.}

To determine the precise role of $\mathrm{Znf1}$ in appressorium maturation, we carried out experiments to determine whether deletion of $Z N F 1$ affected lipid droplet and glycogen mobilization, which are essential prerequisites to appressorium function (Thines et al. 2000). Conidia were placed on hydrophobic surfaces to induce appressorium formation, and lipid body mobilization was observed after Nile Red staining under a confocal laser-scanning

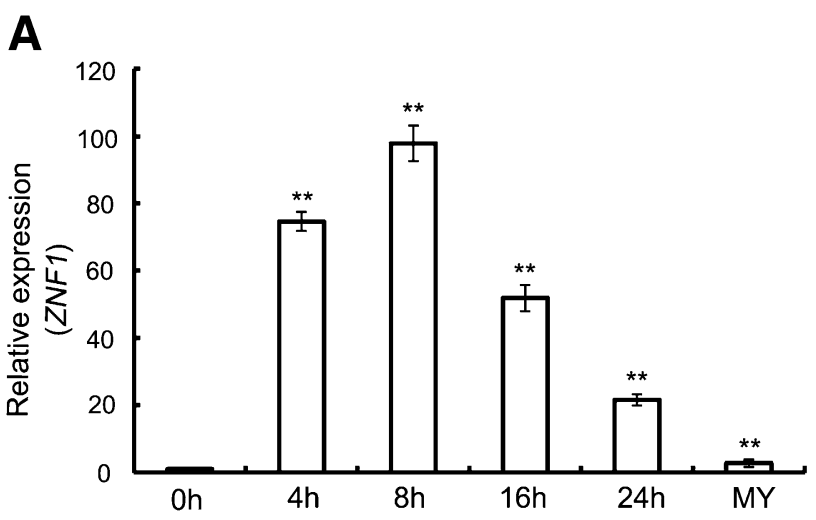

B

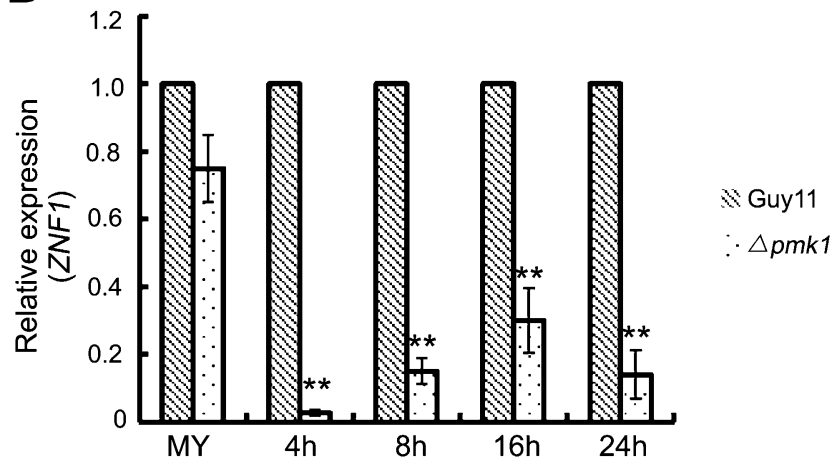

Fig. 6. Expression of ZNF1 in the wild-type strain Guy11 and the $\triangle p m k 1$ mutant during appressorium morphogenesis. A, Quantitative reversetranscription polymerase chain reaction analysis of relative expression of ZNF1 during appressorium development in the wild-type strain Guy11. RNA samples were extracted from mycelium (MY) (2-day-old complete medium liquid culture), conidia, germ tubes, and appressoria $(4,8,16$, and $24 \mathrm{~h}$ incubated on hydrophobic GelBond film) of Guy11. Expression of ZNF1 was significantly elevated during appressorium development. $\mathbf{B}$, Transcriptional expression of ZNF1 during appressoria development in the $\triangle p m k 1$ mutant. The $Z N F 1$ gene exhibited significantly lower levels of expression in $\Delta p m k 1$ mutant during appressorium development at different time intervals $(4,8,16$, and $24 \mathrm{~h})$ compared with that in the wildtype strain. Asterisks indicate a significant difference $(* *, P<0.01)$ microscope. The results showed brighter and larger lipid droplets in conidia and germ tubes of the $\Delta z n f 1-89$ mutant during appressorium formation compared with the wild-type strain, in which lipids gradually disappeared during appressorium maturation (Fig. 8). Even after 48 h, many lipid droplets still remained in conidia and germ tubes of the mutant (Fig. 8). These results indicate that lipid droplet translocation and breakdown occurred more slowly in $\Delta z n f 1$ mutants, consistent with the observed delay in autophagy. The samples were also stained for the presence of glycogen with iodine solution (Thines et al. 2000). Ungerminated conidia of both the wild-type strain Guy11 and the $\Delta z n f 1-89$ mutant were rich in glycogen but glycogen mobilization and degradation was significantly delayed during conidial germination in $\Delta z n f l$ mutants (Supplementary Fig. S8).

We conclude that $\mathrm{Znf1}$ is involved in regulating mobilization and degradation of lipids and glycogen during appressorium formation in $M$. oryzae.

\section{C2H2 zinc-finger domains are essential for proper function of $\mathbf{Z n f 1}$.}

M. oryzae $\mathrm{Znf} 1$ contains four consecutive predicted $\mathrm{C} 2 \mathrm{H} 2$ zinc-finger domains (ZF1 to ZF4) in its $\mathrm{N}$ terminus (Fig. 9A). To determine their function, we constructed alleles in which each of the $\mathrm{C} 2 \mathrm{H} 2$ zinc-finger domains was individually deleted, or where cysteine $(\mathrm{C})$ and histidine $(\mathrm{H})$ residues, which are the key amino acids of $\mathrm{C} 2 \mathrm{H} 2$ zinc-finger domains, were converted into alanine (A) and isoleucine (I), respectively. Each of the constructs was introduced into the $\Delta z n f 1-89$ mutant. The $Z N F 1^{\triangle \mathrm{ZF} 1}$ and $Z N F 1^{\mathrm{C} 18 \mathrm{~A} / \mathrm{H} 36 \mathrm{I}}, Z N F 1^{\triangle \mathrm{ZF} 2}$ and $Z N F 1^{\mathrm{C} 48 \mathrm{~A} / \mathrm{H} 66 \mathrm{I}}$, and $Z N F 1^{\triangle \mathrm{ZF} 3}$ and $Z N F 1^{\mathrm{C} 78 \mathrm{~A} / \mathrm{H} 96 \mathrm{I}}$ mutants were all defective in appressorium formation and pathogenicity (Fig. 9B), indicating that $\mathrm{ZF} 1, \mathrm{ZF} 2$, and $\mathrm{ZF} 3$ domains are necessary for proper function of $\mathrm{Znf} 1$. However, both $Z N F 1^{\triangle \mathrm{ZF} 4}$ and ZNF $1^{\mathrm{C} 108 \mathrm{~A} / \mathrm{H} 126 \mathrm{I}}$ mutants were able to form melanized appressoria and were pathogenic (Fig. 9B), suggesting that the $\mathrm{ZF} 4$ domain is not required for $\mathrm{Znf} 1$ function. We conclude that the $\mathrm{C} 2 \mathrm{H} 2$ zinc-finger domains $\mathrm{ZF} 1, \mathrm{ZF} 2$, and $\mathrm{ZF} 3$ but not $\mathrm{ZF} 4$

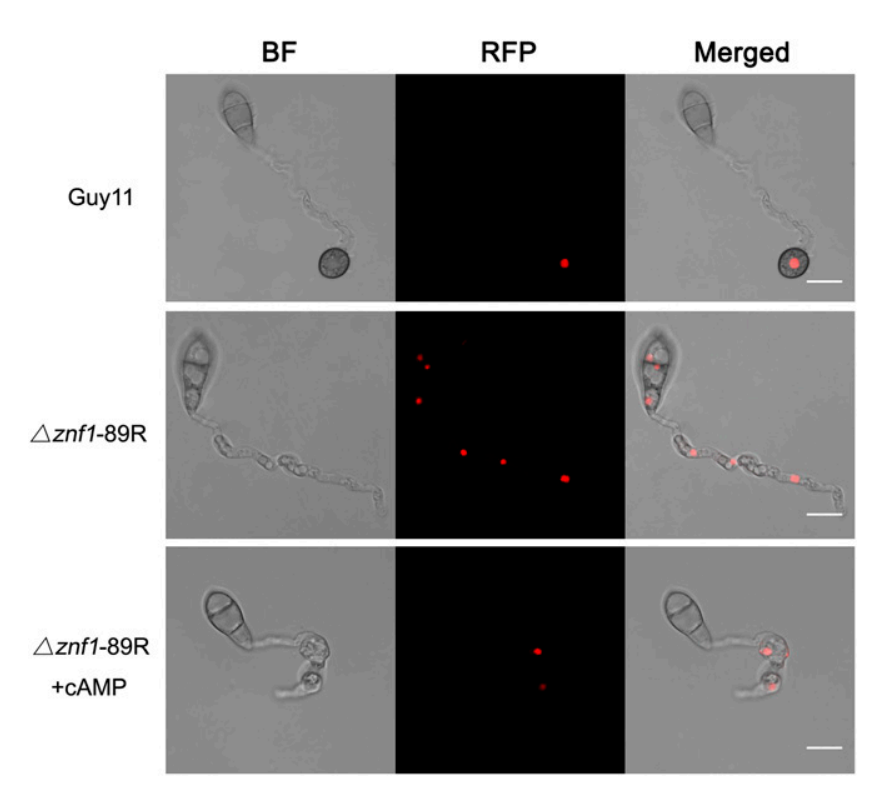

Fig. 7. The $\Delta z n f 1$ mutants are impaired in nuclear degradation during appressorium development. Conidial suspension from $\Delta z n f 1-89 \mathrm{R}$ expressing red fluorescent protein (RFP) was placed on hydrophobic GelBond surfaces. Fluorescence was examined under a confocal laser-scanning microscope at $24 \mathrm{~h}$ after incubation. Exogenous cAMP: $5 \mathrm{mM}$. BF = bright field. Scale bars $=10 \mu \mathrm{m}$ 
are essential for proper function of $\mathrm{Znf1}$ protein in regulating appressorium development in $M$. oryzae.

Bioinformatic analysis showed that $\mathrm{Znf} 1$ contains a predicted nuclear localization signal (NLS), RHRR, at the N terminus (95 to 98 aa) (Fig. 9A). Site-directed mutagenesis to delete this signal was carried out and the resulting allele was transformed into the $\triangle z$ zfl-89 mutant. The $Z N F 1^{\triangle \mathrm{RHRR}}$ mutants were unable to form appressoria and remained defective in plant infection (Fig. 9B), indicating that the putative NLS is essential for Znf1 function in $M$. oryzae.

To further characterize the function of Znf1, subcellular localization of Znf1 protein was investigated using GFP tagging. First, we constructed a vector expressing ZNF1-GFP (C-terminal GFP tagging) driven by a $1.6-\mathrm{kb}$ native promoter (Supplementary Fig. S9A). The construct was able to complement all phenotypic defects in appressorium formation and pathogenicity of the $\Delta z n f 1-89$ mutant. Surprisingly, however, no green fluorescence was observed in hyphae, conidia, germ tubes, and appressoria of these strains (data not shown). Therefore, four other vectors were constructed. Similarly, the two constructs, native promoter-GFP-ZNF1 (N-terminal tagging) and TrpC-GFP$Z N F 1$, were able to complement the phenotypic defects of the $\Delta z n f 1-89$ mutant but no GFP fluorescence was observed. For construct IV, GFP fluorescence was mainly observed in the cytoplasm. Because Znf1 contains a predicted NLS in its $\mathrm{N}$ terminus, a DNA fragment containing the native promoter and the partial coding sequence (encoding 138 aa of the Znf1 N-terminal domain) was ligated with GFP. For this construct (V), green fluorescence was observed in the nucleus of hyphae, conidia, and germ tubes of transformants (Supplementary Fig. S9B), indicating that the NLS of Znf1 plays an important role in directing Znf1 subcellular localization. Moreover, GFP fluorescence was

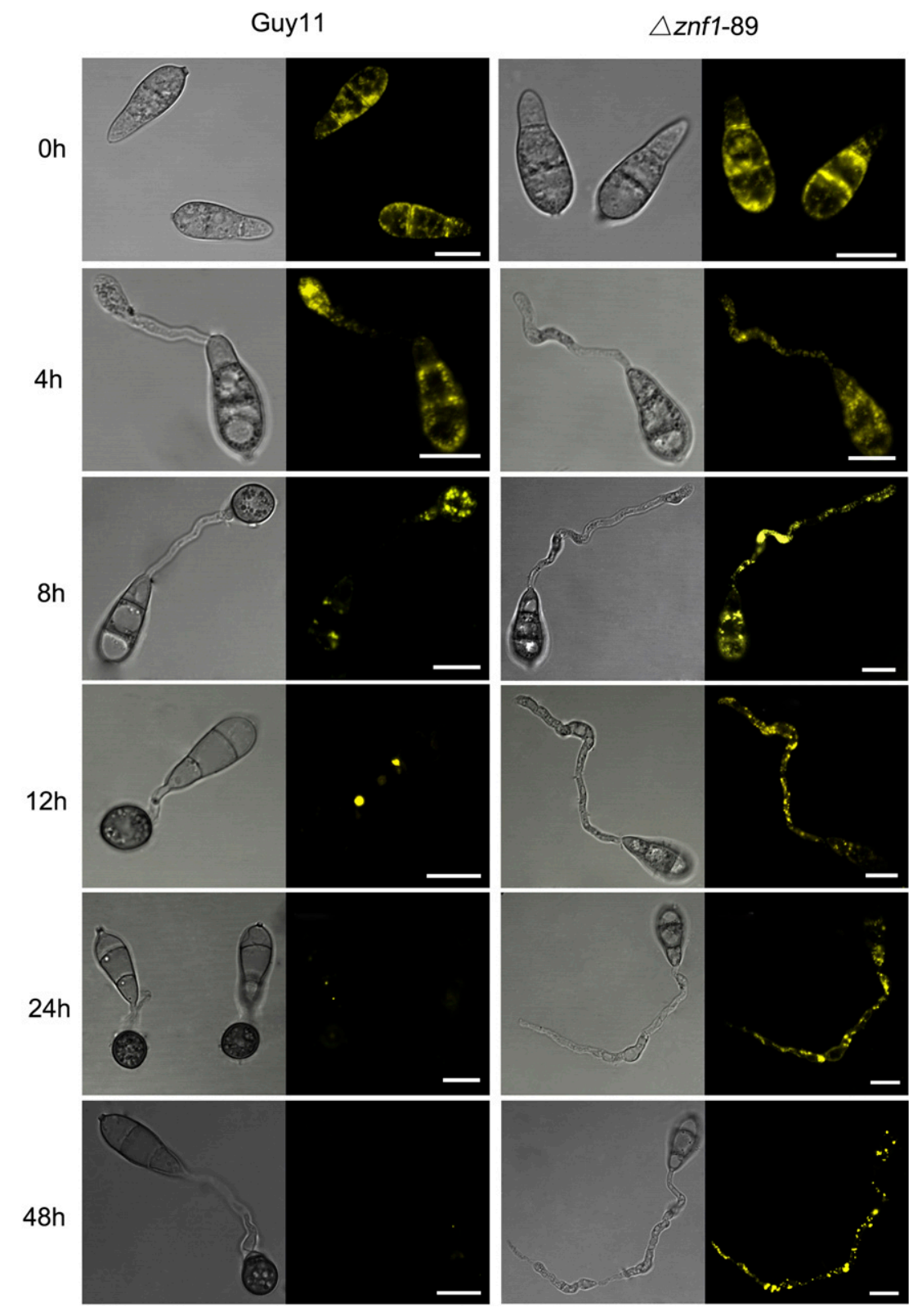

Fig. 8. Lipid body movement of Magnaporthe oryzae Guy11 and $\Delta z$ fn1 mutants during appressorium morphogenesis. Conidia of the wild-type strain Guy 11 and the $\Delta z n f 1-89$ mutant were incubated in water droplets on the hydrophobic GelBond film surfaces and allowed to form appressoria for $48 \mathrm{~h}$. Samples at different time intervals $(0,4,8,12,24$, and $48 \mathrm{~h}$ ) were stained with Nile Red to show the presence of lipid bodies by epifluorescence microscopy. Lipid bodies of Guy11 migrated to the tips of germ tubes and young appressoria and disappeared during the maturation of appressoria. The $\Delta z n f 1-89$ mutant showed a delay in lipid mobilization and degradation and, even at 24 or $48 \mathrm{~h}$, many lipid drops still presented in conidia and germ tubes. Scale bars $=10 \mu \mathrm{m}$. 
much brighter in germ tubes at 4 and $8 \mathrm{~h}$ than in conidia at $0 \mathrm{~h}$, which is consistent with the results of qRT-PCR (Fig. 6A).

\section{DISCUSSION}

In this study, we identified a nonpathogenic mutant XF696 by screening a T-DNA insertional mutagenesis library. Analysis of the mutation confirmed a single T-DNA insertion within the promoter of $Z N F 1$, which encodes a putative $\mathrm{C} 2 \mathrm{H} 2$ zinc-finger TF (zinc-finger protein 32). Targeted gene deletion mutants of ZNF1 were unable to cause blast disease on susceptible barley and rice, even when plant surfaces were wounded (Fig. 2). Znf1 shared only $13.10 \%$ amino acid identity with human Znf32. In humans, the ZNF32 gene, a 273-aa protein with seven consecutive $\mathrm{C} 2 \mathrm{H} 2$ zinc-finger motifs, has been mapped to a human chromosome region on 10q23-24 (Cannizzaro et al. 1993) and is involved in cell differentiation and tumor development, although its precise function is unknown (Wei et al. 2012). Bioinformatic analysis revealed that Znf1-like proteins are present in several filamentous fungi, including Gaeumannomyces graminis, Verticillium dahliae, and $C$. graminicola. However, we also observed that $M$. oryzae $\mathrm{Znf1}$ is highly distinct from the most similar genes in other fungal species such as F. graminearum FGSG_08617 (GzC2H066), Aspergillus nidulans ANID_05583 (As1A), and N. crassa NCU03421, with amino acid sequence identity of only $12.91,22.04$, and $29.19 \%$, respectively. This is consistent with phylogenetic analysis, which showed that Znf1 forms a separate clade
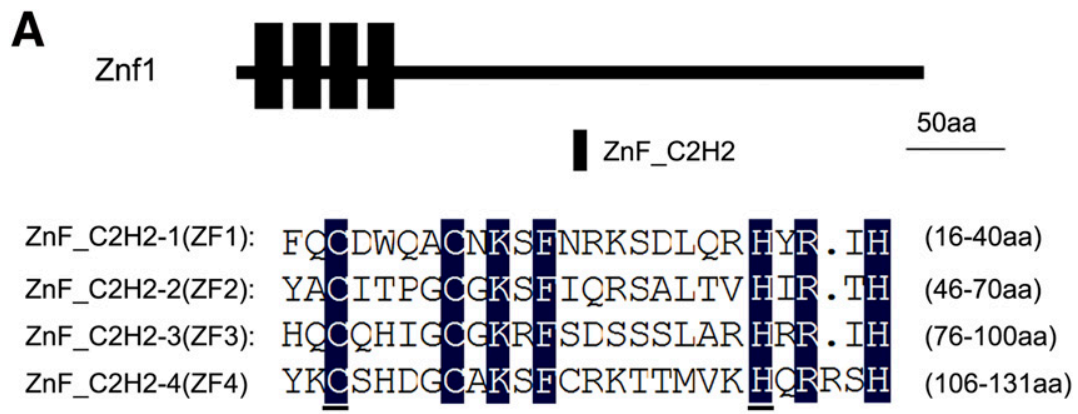

Nuclear localization signal (NLS): RHRR(95-98aa)

B
Strains
Appressorium Formation
Plant Infection

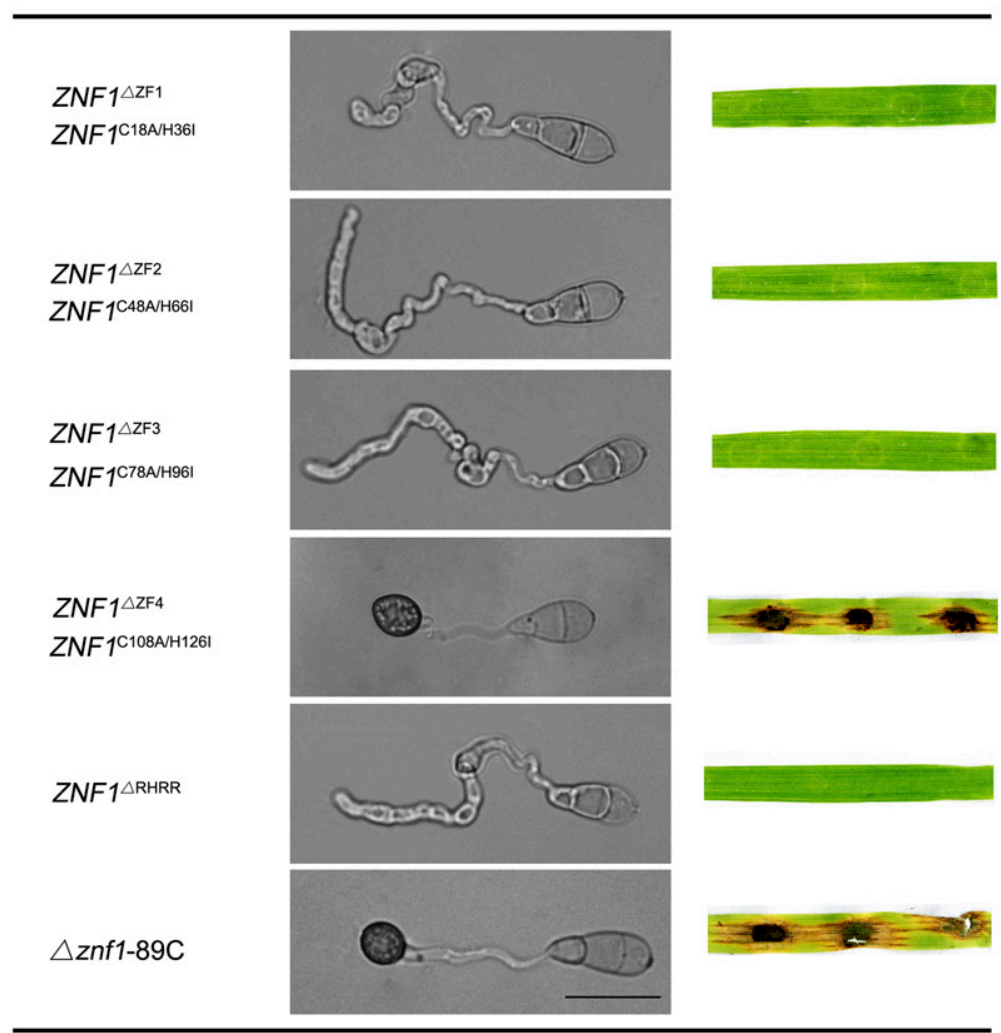

Fig. 9. Functional analysis of predicted domains in the Znf1 protein. A, Position and sequence of the deleted four predicted zinc-finger domains (ZF1, ZF2, ZF3, and ZF4) and a nuclear localization signal (NLS) in Znf1 protein. Amino acid (aa) residues, which were mutated by site-directed mutagenesis, in the zinc fingers are underlined. B, Functional analysis of the domains in Znf1. Each of the three zinc-finger domains ZF1, ZF2, and ZF3 was required for the full function of Znf1. Similarly, mutation of the key amino acid residues in the zinc-finger domains, in which histidine (H) and cysteine (C) residues were substituted with isoleucine (I) and alanine (A), respectively, resulted in defects in appressorium formation and plant infection. However, ZF4 was not required for the function of Znf1. The predicted NLS (RHRR) was also crucial for the function of Znf1 protein. The $\Delta z n f 1-89 \mathrm{C}$ was a complemented strain. Scale bar $=$ $10 \mu \mathrm{m}$. 
with the Znf1-like proteins from $G$. graminis, V. dahliae, and C. graminicola. There has clearly been considerable divergence in function across this group of putative TF in filamentous fungi. Recently, it has been shown that deletion of $\mathrm{GzC} 2 \mathrm{HO} 66$ resulted in defects in sexual development, mycotoxin production, and virulence in F. graminearum (Guo et al. 2015; Son et al. 2011). By contrast, in A. nidulans, AslA is required for vacuole-mediated tolerance to $\mathrm{K}^{+}$stress (Park et al. 2015) and, in N. crassa, NCU03421 is essential for normal hyphal growth (Tian et al. 2011). We conclude that there is considerable variation in Znf1-related proteins among filamentous fungi but that they all appear to be developmental regulators, albeit acting at very different stages of fungal development.

We observed that $M$. oryzae ZNF1 had two different spliced isoforms, S I and S II, in vivo (Fig. 1E). The only difference between the two variants is that a 127-bp DNA fragment at the $\mathrm{C}$ terminus of the coding sequence was removed in the S II isoform. Initially, we suspected this difference of the isoforms could be caused by a sequencing error. However, further sequencing data showed that both of the two spliced variants appeared in mycelium, conidia, germinated conidia ( $4 \mathrm{~h}$ after incubation), and appressoria (24 h), although S I was the predominant splice variant $(73.13 \%)$. We found that S I but not S II was able to complement all $\Delta z n f 1$ mutant phenotypes (Fig. 1F). The lack of function of the S II splice variant is likely caused by changes of coding sequence in its $\mathrm{C}$ terminus. In addition, we found that both S I and S II could be amplified from mRNA extracted from $M$. oryzae $70-15$ by RT-PCR, indicating that the two splice isoforms are presented in different strains of M. oryzae (data not shown). However, why this apparently nonfunctional spliced variant (S II) exists so commonly in all fungal tissues tested at different developmental stages is unclear, because no phenotypes could be attributed to its specific loss.

Like most fungal pathogens, asexual reproduction and appressorium formation play key roles in the disease cycle of M. oryzae (Talbot 2003; Wilson and Talbot 2009). To date, transcriptional regulators, which positively regulate conidiation, have been identified, including Con7 (Odenbach et al. 2007), Mig1 (Mehrabi et al. 2008), Cos1 (Zhou et al. 2009), Crz1 (Zhang et al. 2009), Mstu1 (Nishimura et al. 2009), Ap1 (Guo et al. 2011), Hox2/Htf1 (Kim et al. 2009; Liu et al. 2010), Ldb1 (Li et al. 2010a), Som1 and Cdtf1 (Yan et al. 2011), and COD1 and COD2 (Chung et al. 2013). Here, by contrast, we demonstrated that $M$. oryzae $\mathrm{Znf1}$ may act as a negative regulator of asexual sporulation. The $\Delta z n f l$ mutants, for example, produced significantly more conidia compared with the wildtype strain (Fig. 3C), while qRT-PCR analysis revealed that the expression level of COS1 was significantly upregulated in $\Delta z$ ffl mutants (Fig. 3D). Because $M$. oryzae Cos1 is a key TF that positively regulates conidiophore production, the increase in conidial production of $\Delta z n f 1$ mutants may be a consequence of more conidiophore development by upregulation of COSI gene expression. Interestingly, ZNF1 was dispensable for vegetative growth, pigment production, and sexual reproduction in $M$. oryzae, suggesting that its roles in sporulation and appressorium morphogenesis are specific functions and not merely a consequence of poor growth or fitness.

It is clear that Znf1 is an important regulator of appressorium development, a key step in the infection cycle of the fungus (Talbot 2003; Wilson and Talbot 2009). The ZNF1 gene is significantly upregulated during appressorium development (Fig. 6A) and ZNF1 deletion mutants fail to develop melanized appressoria, although they were able to initiate the swelling of hyphal tips in response to surface hydrophobicity or the presence of cAMP (Figs. 4 and 5). Therefore, M. oryzae Znf1 plays an essential role in regulating appressorium-mediated plant infection.
Interestingly, $\Delta z n f 1$ mutants were also unable to proliferate within host cells and, in this way, the mutant phenotype strongly resembles those of the Pmk1-MAPK mutant (Xu and Hamer 1996; Zhao et al. 2005). Consistent with this observation, the expression of $Z N F 1$ during appressorium development was significantly suppressed in $\triangle p m k 1$ mutants (Fig. 6B). Moreover, deletion of the ZNF1 gene results in similar effects on mitosis to the absence of $P M K 1$ (Fig. 7). A recent global analysis of the transcription profile of $M$. oryzae during appressorium development also confirmed that ZNF1 is downregulated in $\triangle p m k 1$ mutants (Soanes et al. 2012). When considered together, these data suggest that ZNF1 may be indirectly regulated by Pmk1 to serve a role in the regulation of appressorium development and invasive growth in M. oryzae.

In $M$. oryzae, glycogen reserves and lipid bodies have previously been shown to move into appressoria prior to turgor generation (Bhambra et al. 2006; Thines et al. 2000; Wang et al. 2007). This mobilization process is controlled by the Pmk1MAPK pathway (Zhao et al. 2005), while lipid and glycogen breakdown in appressoria are controlled by the cAMP-PKA signaling pathway (Thines et al. 2000). Recently, it has been reported that glycogen metabolic genes, including $A G L 1$ and GPH1, which encode amyloglucosidase and glycogen phosphorylase, respectively, play an important role in plant infection by $M$. oryzae (Badaruddin et al. 2013). We observed that mobilization and degradation of both glycogen and lipid reserves were impaired in $\Delta z n f l$ mutants during appressorium differentiation (Fig. 8). Given that the transcriptional expression of ZNFl was downregulated in $\Delta p m k 1, \Delta m a c l$, and $\triangle c p k A$ mutants (Fig. 6B), it seems likely that Znf1 acts downstream of the Pmk1-MAPK and cAMP-PKA response pathways to regulate appressorium maturation, including mobilization and degradation of lipid and glycogen reserves. The developmental defects of the $\Delta z n f 1$ mutant also affected infection-associated autophagy, which showed a delay and did not result in conidial cell death, which normally occurs during appressorium development. This is also consistent with the effect on nuclear degradation, suggesting that $\mathrm{Znf} 1$ plays a significant role in the appressorium development pathway, which required cell cycle progression, autophagic cell death, and mobilization of lipid and glycogen reserves from the collapsing conidium to the incipient appressorium to allow maturation and plant infection.

Znf1 is a typical $\mathrm{C} 2 \mathrm{H} 2$ zinc-finger $\mathrm{TF}$ and contains four predicted $\mathrm{C} 2 \mathrm{H} 2$ zinc-finger domains ( $\mathrm{ZF} 1$ to $\mathrm{ZF} 4)$ and one potential NLS (RHRR) at its $\mathrm{N}$ terminus (Fig. 9A). Sitedirected mutagenesis and phenotypic analysis confirmed that each of the ZF1, ZF2, ZF3, and NLS domains but not ZF4 is essential for proper function (Fig. 9B). In this study, however, we were not able to observe GFP fluorescence following expression of either C-terminal or N-terminal GFP fusions of $Z N F 1$, even though all phenotypes of the $\Delta z n f 1-89$ mutant could be complemented. RT-PCR analysis showed that the GFP gene was transcriptionally expressed and Western blotting experiments with anti-GFP antibody also detected a 70-kDa band (data not shown), indicating that GFP was successfully expressed in these complemented strains. It is possible that a conformational change in the GFP-fusion proteins, therefore, may lead to lack of fluorescence. This explanation was partially supported by the fact that GFP fluorescence was observed when truncated versions of the gene fusion (vector IV or V) were expressed in the $\Delta z n f 1-89$ mutant and nuclear localization was also observed. Moreover, mutagenesis revealed that the NLS of Znf1 was essential for its function (Fig. 9B). Therefore, it is likely that, as expected, the NLS of Znf1 plays an important role in directing the protein to the nucleus.

In summary, we conclude that $\mathrm{Znf} 1$ is a $\mathrm{C} 2 \mathrm{H} 2$ zinc-finger $\mathrm{TF}$ that is essential for the regulation of appressorium development 
and pathogenicity by the rice blast fungus $M$. oryzae. It appears to operate downstream of the Pmk1-MAPK pathway and the cAMP response pathway and serve a role in controlling the physiological changes that accompany appressorium maturation, including autophagy, mobilization of lipid bodies and glycogen, and the maturation of appressoria. In order to define the subset of genes regulated by Znf1 in more detail, it will be necessary to carry out comparative transcriptional profiling with mutants lacking the Pmk1 MAPK, in order to see whether the precise subset of genes can be determined hierarchically, by comparison with the wider transcriptional profile associated with loss of Pmk1 (Soanes et al. 2012). The fact that the $\Delta z n f 1$ mutants also sporulate profusely suggests that $\mathrm{Znf} 1$ is a negative regulator of conidiation, which might suggest a role in the transition from polarized hyphal growth to determinate, yeast-like, isotropic expansion of cells, which occurs during conidiogenesis and during appressorium formation. In this way, it will be interesting to observe whether the cytoskeletal changes associated with appressorium morphogenesis are also perturbed by the absence of $\mathrm{Znf1}$, as seems likely based on its mutant phenotypes. The mutant will also provide a valuable tool in which to study the parallels between conidiogenesis and initiation of appressorium development, which are clearly related developmental processes that may share regulatory components in $M$. oryzae.

\section{MATERIALS AND METHODS}

\section{Strains, culture conditions, and molecular manipulations of nucleic acids.}

All mutants described in the present study were generated from M. oryzae wild-type strain Guy11 (Leung et al. 1988). Standard growth and storage procedures for fungal strains were performed, as described previously (Talbot et al. 1993). Agrobacterium tumefaciens AGL1 was used for T-DNA insertional transformation. Escherichia coli strain DH-5 $\alpha$ was used for routine bacterial transformations and maintenance of various plasmids in this study. Southern blot analysis was performed by the digoxigenin high prime DNA labeling and detection starter Kit II (Roche). General procedures for nucleic acid analysis followed standard protocols (Sambrook et al. 1989). Total RNA was extracted from mycelium of $M$. oryzae using the RNAiso Plus reagent (TaKaRa), according to the manufacturer's instructions.

\section{T-DNA insertional mutagenesis.}

A. tumefaciens-mediated transformation and construction of the T-DNA insertional mutagenesis library followed a procedure described previously ( $\mathrm{Li}$ et al. 2010b).

\section{Construction of vectors and fungal transformation.}

To construct $Z N F 1$ gene replacement vector pKO-696, a 3.7-kb genomic DNA sequence containing a $1.65-\mathrm{kb}$ ZNF1 ORF, approximately $1.0 \mathrm{~kb}$ upstream and approximately $1.0 \mathrm{~kb}$ downstream flanking sequences of the gene locus were amplified using primer pairs of $1 \mathrm{~F} / 2 \mathrm{R}$ and cloned sequentially into pGEM-T easy vectors to generate pT-696. The 1.4-kb $H P H$ gene cassette, which encodes hygromycin phosphotransferase under control of the Aspergillus nidulans TrpC promoter (Carroll et al. 1994), was amplified with primers 696-Hph-F and 696-Hph-R using pCB1003 as a template and clone into pGEM-T easy vectors. The resulting vector was digested with BamHI and the released fragment was inserted into dephosphorylated pT-696 with the same digestions to generate pKO696. The pKO-696 vector was use to transform into M. oryzae Guy11 protoplasts to generate gene null mutants, as previously described (Talbot et al. 1993).

To construct complementation vector pHB-696, a 3.22-kb fragment, including a $1.65-\mathrm{kb}$ full-length ZNF1 gene coding sequence and a $1.57-\mathrm{kb}$ promoter region, was amplified using primers $\mathrm{HB}-\mathrm{F}$ and $\mathrm{HB}-\mathrm{R}$ and then cloned into pGEM-T easy to produce pGEM-HB. The pGEM-HB was double digested with SpeI and $\mathrm{XbaI}$ and the resulting fragment was inserted into the corresponding site of pCB1532 to create pHB696. The plasmid pHB-696 was used to transform $\Delta z n f 1-89$ and XF696 mutants. Transformants carrying a single insertion were selected and complementation of the mutant phenotypes was analyzed.

\section{Deletion of $\mathrm{C} 2 \mathrm{H} 2$ zinc-finger domains and site-directed mutagenesis in ZNF1.}

For generating the $Z N F 1^{\triangle \mathrm{ZF} 1}, Z N F 1^{\triangle \mathrm{ZF} 2}, Z N F 1^{\triangle \mathrm{ZF} 3}$, and $Z N F 1^{\triangle \mathrm{ZF} 4}$ mutants, the $\mathrm{C} 2 \mathrm{H} 2$ zinc-finger domain deletion vectors $\mathrm{pZF} 1, \mathrm{pZF} 2, \mathrm{pZF} 3$, and $\mathrm{pZF} 4$, respectively, were constructed by double-joint PCR (Yu et al. 2004). To construct pZF1, 1.6- and 1.3-kb fragments were first amplified with the primer pairs HB-F1/ZF1-R and ZF1-F/HB-R1, respectively, using pHB-696 as a template. Both primers ZF1-R and ZF1-F were especially designed to delete the $\mathrm{ZF} 1$ domain. Then, the two PCR products were mixed and PCR was performed (10 reaction cycles) without adding primers. Using the mixture as a template, a $2.9-\mathrm{kb}$ fragment containing a $1.57-\mathrm{kb}$ native promoter and a 1.33-kb ZNF1 gene-coding sequence (without ZF1 domain) was amplified with the primers HB-F1 and HB-R1. Finally, according to the manufacturer's instructions for the One-Step Cloning Kit, the 2.9-kb fragment was cloned into pCB1532 to create pZF1, which was subsequently transformed into $\triangle z n f 1$ mutants to generate $Z N F 1^{\triangle Z F 1}$ mutants. The other mutants $\left(Z N F 1^{\triangle \mathrm{ZF} 2}, Z N F 1^{\triangle \mathrm{ZF} 3}\right.$, and $\left.Z N F 1^{\triangle \mathrm{ZF} 4}\right)$ were obtained using a similar strategy. The primers used for constructing these mutants were shown in Supplementary Table S1.

For generating $\mathrm{ZNF1}^{\mathrm{C} 18 \mathrm{~A} / \mathrm{H} 36 \mathrm{I}}, \mathrm{ZNF1}{ }^{\mathrm{C} 48 \mathrm{~A} / \mathrm{H} 66 \mathrm{I}}, \mathrm{ZNF1}{ }^{\mathrm{C} 78 \mathrm{~A} / \mathrm{H} 96 \mathrm{I}}$, and $Z N F 1^{\mathrm{C} 108 \mathrm{~A} / \mathrm{H} 126 \mathrm{I}}$ mutants, the site-directed mutagenesis vectors $\mathrm{pC} 18 \mathrm{~A} / \mathrm{H} 36 \mathrm{I}, \mathrm{pC} 48 \mathrm{~A} / \mathrm{H} 66 \mathrm{I}, \mathrm{pC} 78 \mathrm{~A} / \mathrm{H} 96 \mathrm{I}$, and $\mathrm{pC} 108 \mathrm{~A} / \mathrm{H} 126 \mathrm{I}$, respectively, were constructed as described below. For construction of pC18A/H36I, site-directed mutagenesis introduced $\mathrm{C} 18 \mathrm{~A}-\mathrm{R}$ and $\mathrm{C} 18 \mathrm{~A}-\mathrm{F}$ to substitute cysteine $(\mathrm{C})$ with alanine $(\mathrm{A})(\mathrm{TGC} \rightarrow \mathrm{GCC})$ and H36I-R/H36I$\mathrm{F}$ to substitute histidine $(\mathrm{H})$ with isoleucine $(\mathrm{I})(\mathrm{CAT} \rightarrow \mathrm{ATC})$ in ZF1 domain. The pC18A/H36I vector was constructed by double-joint PCR and One-Step Cloning, as described above. A similar strategy was used for the construction of the other site-directed mutagenesis vectors. The primers used for constructing these vectors were shown in Supplementary Table S1.

\section{Pathogenicity assays.}

For cut-leaf assays, leaf fragments were cut from 7-day-old barley (Golden Promise) or 2-week-old rice (CO-39) seedlings and placed in plastic plates containing wetted filters. CM cultural plugs containing mycelium or conidial suspensions $(1 \times$ $10^{5}$ conidia $\mathrm{ml}^{-1}$ ) were placed onto leaf sections and the plates were incubated at $25^{\circ} \mathrm{C}$. Wounded leaves were prepared by removing the surface cuticle by abrasion with an emery board, as described previously ( $\mathrm{Li}$ et al. 2010a; Wang et al. 2007). For spray-inoculation assays, conidial suspensions with $5 \times 10^{4}$ conidia ml $\mathrm{ml}^{-1}$ diluted in $0.05 \%$ Tween- 20 were spray inoculated using an artist's airbrush onto barley and rice plants. Inoculated seedlings were kept in black plastic bags for $24 \mathrm{~h}$ to maintain high humidity and then transferred to controlled-environment chambers at $25^{\circ} \mathrm{C}$ and $90 \%$ relative humidity. For root infection assays, rice seed were germinated for 3 days at $28^{\circ} \mathrm{C}$ and then transferred to plastic plates contained $2 \%$ water agar. Mycelial plugs were carefully placed on rice roots. Each test was repeated three times. Disease lesions were examined and photographed after 5 days of incubation. 


\section{Analysis of fungal growth, sporulation, appressorium formation, and genetic crosses.}

Vegetative growth was assessed by measurement of colony diameters in plate cultures of $M$. oryzae grown on $\mathrm{CM}$ at $25^{\circ} \mathrm{C}$ for 10 days. Conidiogenesis was analyzed by harvesting conidia from the surface of 10-day-old plate cultures and determining the concentration of the resulting conidial suspensions using a hemocytometer. For appressorium formation and penetration assays, $20 \mu$ of conidial suspensions of $1 \times 10^{4}$ spores $\mathrm{ml}^{-1}$ were dropped onto hydrophobic GelBond film and onion or barley epidermis surfaces and incubated at $25^{\circ} \mathrm{C}$. For appressorium formation from the tips of hyphae, mycelia of various strains were harvested from 48-h liquid CM cultures, and the mycelium fragment suspensions were placed on hydrophobic GelBond surfaces to allow appressorium development. Fertility assays were carried out by pairing tested strains (MAT1-2) with standard tester strain TH3 (MAT1-1) on oatmeal agar plates, as described previously ( $\mathrm{Li}$ et al. 2010a). Each test was repeated three times.

\section{qRT-PCR analysis.}

qRT-PCR was performed according to the guidelines for minimum information for publication of quantitative real-time PCR experiments (MIQE). Total RNA was isolated from mycelium using RNAiso Plus reagent (TaKaRa) and used to synthesize first-strand cDNA using PrimeScriptRT (TaKaRa). qRT-PCR was performed with GoTag qPCR Master Mix (Promega Corp.) using the Bio-Rad CFX96 Real-Time System. Primers used for qRT-PCR assays were listed in Supplementary Table S1. The relative expression level of each gene was calculated as the $2^{-\Delta \Delta C T}$ method (Livak and Schmittgen 2001) with $\beta$-tubulin (MGG_00604) as reference. Mean and standard deviation were determined with data from three or more replicates.

\section{Y2H assay.}

The $\mathrm{Y} 2 \mathrm{H}$ assay was performed according to the BD Matchmaker Library Construction \& Screening Kits instructions (Clontech). Full-length cDNA of each candidate gene were amplified with primers listed in Supplementary Table S1. For the autoactivation assay of Znf1, the ZNF1 cDNA was cloned into bait plasmid pGBK. The resulting bait vector and empty prey vector were cotransformed into yeast strain AH109. For the interaction assay, the ZNF1 cDNA and PMK1 cDNA were cloned into prey plasmid $\mathrm{pGAD}$ and bait plasmid pGBK, respectively. Growth of yeast transformants was determined on SD-Trp-Leu-His-Ade medium.

\section{Mobilization of lipid bodies and glycogen during appressorium development.}

Conidial suspensions of various tested $M$. oryzae strains were incubated on hydrophobic GelBond and incubated for appressorium formation at $25^{\circ} \mathrm{C}$. Lipid droplets in the germinating conidia, germ tubes, and appressoria were visualized by staining with Nile Red solution consisting $50 \mathrm{mM}$ Tris-maleate buffer (pH 7.5), polyvinyl pyrrolidone at $20 \mathrm{mg} \mathrm{ml}^{-1}$, and Nile Red Oxazone at $2.5 \mu^{-1} \mathrm{ml}^{-1}$ (Thines et al. 2000; Weber et al. 1999). Microscopy was performed using a Zeiss Lsm 780 inverted confocal laser-scanning microscope at intervals. Glycogen deposits within developing appressoria were visualized using iodine solution, consisting of KI at $60 \mathrm{mg} \mathrm{ml}^{-1}$ and $\mathrm{I}_{2}$ at $10 \mathrm{mg} \mathrm{ml}^{-1}$ in distilled water (Weber et al. 1998). After a few seconds of staining, glycogen deposits were observed microscopically.

\section{ACKNOWLEDGMENTS}

This work was supported by the National Key Basic Research and Development Program of China (2012CB114002), the Program for Changjiang
Scholars and Innovative Research Team in University (IRT0943), and the Natural Science Foundation of China (grant number 31370172) to Z. Wang.

\section{LITERATURE CITED}

Badaruddin, M., Holcombe, L. J., Wilson, R. A., Wang, Z. Y., Kershaw, M. J., and Talbot, N. J. 2013. Glycogen metabolic genes are involved in trehalose-6-phosphate synthase-mediated regulation of pathogenicity by the rice blast fungus Magnaporthe oryzae. PLoS Pathog. 9:e1003604.

Bhambra, G. K., Wang, Z. Y., Soanes, D. M., Wakley, G. E., and Talbot, N. J. 2006. Peroxisomal carnitine acetyl transferase is required for elaboration of penetration hyphae during plant infection by Magnaporthe grisea. Mol. Microbiol. 61:46-60.

Cannizzaro, L. A., Aronson, M. M., and Thiesen, H. J. 1993. Human zinc finger gene ZNF23 (Kox16) maps to a zinc finger gene cluster on chromosome 16q22, and ZNF32 (Kox30) to chromosome region 10q23q24. Hum. Genet. 91:383-385.

Carroll, A. M., Sweigard, J. A., and Valent, B. 1994. Improved vectors for selecting resistance to hygromycin. Fungal Genet. Newsl. 41:22.

Choi, J., Kim, Y., Kim, S., Park, J., and Lee, Y. H. 2009. MoCRZ1, a gene encoding a calcineurin-responsive transcription factor, regulates fungal growth and pathogenicity of Magnaporthe oryzae. Fungal Genet. Biol. 46:243-254.

Choi, W., and Dean, R. A. 1997. The adenylate cyclase gene MAC1 of Magnaporthe grisea controls appressorium formation and other aspects of growth and development. Plant Cell 9:1973-1983.

Chumley, F. G., and Valent, B. 1990. Genetic analysis of melanin-deficient, nonpathogenic mutants of Magnaporthe grisea. Mol. Plant-Microbe Interact. 3:135-143.

Chung, H., Choi, J., Park, S. Y., Jeon, J., and Lee, Y. H. 2013. Two conidiation-related $\mathrm{Zn}(\mathrm{II}) 2 \mathrm{Cys} 6$ transcription factor genes in the rice blast fungus. Fungal Genet. Biol. 61:133-141.

de Jong, J. C., McCormack, B. J., Smirnoff, N., and Talbot, N. J. 1997. Glycerol generates turgor in rice blast. Nature 389:244-245.

Guo, L., Breakspear, A., Zhao, G., Gao, L., Kistler, H. C., Xu, J.-R., and Ma, L.-J. 2015. Conservation and divergence of the cyclic adenosine monophosphate-protein kinase A (cAMP-PKA) pathway in two plant pathogenic fungi: Fusarium graminearum and F. verticillioides. Online publication. Mol. Plant Pathol. doi:10.1111/mpp.12272

Guo, M., Chen, Y., Du, Y., Dong, Y., Guo, W., Zhai, S., Zhang, H., Dong, S., Zhang, Z., Wang, Y., Wang, P., and Zheng, X. 2011. The bZIP transcription factor MoAP1 mediates the oxidative stress response and is critical for pathogenicity of the rice blast fungus Magnaporthe oryzae. PLoS Pathog. 7:e1001302.

Howard, R. J., Ferrari, M. A., Roach, D. H., and Money, N. P. 1991. Penetration of hard substrates by a fungus employing enormous turgor pressures. Proc. Natl. Acad. Sci. U.S.A. 88:11281-11284.

Kershaw, M. J., and Talbot, N. J. 2009. Genome-wide functional analysis reveals that infection-associated fungal autophagy is necessary for rice blast disease. Proc. Natl. Acad. Sci. U.S.A. 106:15967-15972.

Kim, S., Park, S. Y., Kim, K. S., Rho, H. S., Chi, M. H., Choi, J., Park, J., Kong, S., Park, J., Goh, J., and Lee, Y. H. 2009. Homeobox transcription factors are required for conidiation and appressorium development in the rice blast fungus Magnaporthe oryzae. PLoS Genet. 5:e1000757.

Kong, L. A., Li, G. T., Liu, Y., Liu, M. G., Zhang, S. J., Yang, J., Zhou, X. Y., Peng, Y. L., and Xu, J. R. 2013. Differences between appressoria formed by germ tubes and appressorium-like structures developed by hyphal tips in Magnaporthe oryzae. Fungal Genet. Biol. 56:33-41.

Lee, Y. H., and Dean, R. A. 1993. cAMP regulates infection structure formation in the plant pathogenic fungus Magnaporthe grisea. Plant Cell 5:693-700.

Leung, H., Borromeo, E. S., Bernardo, M. A., and Notteghem, J. L. 1988. Genetic analysis of virulence in the rice blast fungus Magnaporthe grisea. Phytopathology 78:1227-1233.

Li, Y., Liang, S., Yan, X., Wang, H., Li, D., Soanes, D. M., Talbot, N. J., Wang, Z., and Wang, Z. 2010a. Characterization of MoLDB1 required for vegetative growth, infection-related morphogenesis, and pathogenicity in the rice blast fungus Magnaporthe oryzae. Mol. Plant-Microbe Interact. 23:1260-1274

Li, Y., Yan, X., Wang, H., Liang, S., Ma, W. B., Fang, M. Y., Talbot, N. J., and Wang, Z. Y. 2010b. MoRic8 Is a novel component of G-protein signaling during plant infection by the rice blast fungus Magnaporthe oryzae. Mol. Plant-Microbe Interact. 23:317-331.

Liu, W., Xie, S., Zhao, X., Chen, X., Zheng, W., Lu, G., Xu, J. R., and Wang, Z. 2010. A homeobox gene is essential for conidiogenesis of the rice blast fungus Magnaporthe oryzae. Mol. Plant-Microbe Interact. 23: 366-375. 
Liu, Y. G., and Chen, Y. 2007. High-efficiency thermal asymmetric interlaced PCR for amplification of unknown flanking sequences. Biotechniques 43:649-656.

Livak, K. J., and Schmittgen, T. D. 2001. Analysis of relative gene expression data using real-time quantitative PCR and the 2(-delta delta C(T)) method. Methods 25:402-408.

Mehrabi, R., Ding, S., and Xu, J. R. 2008. MADS-box transcription factor mig1 is required for infectious growth in Magnaporthe grisea. Eukaryot. Cell 7:791-799.

Mitchell, T. K., and Dean, R. A. 1995. The cAMP-dependent protein kinase catalytic subunit is required for appressorium formation and pathogenesis by the rice blast pathogen Magnaporthe grisea. Plant Cell 7: 1869-1878.

Nishimura, M., Fukada, J., Moriwaki, A., Fujikawa, T., Ohashi, M., Hibi, T., and Hayashi, N. 2009. Mstu1, an APSES transcription factor, is required for appressorium-mediated infection in Magnaporthe grisea. Biosci. Biotechnol. Biochem. 73:1779-1786.

Odenbach, D., Breth, B., Thines, E., Weber, R. W., Anke, H., and Foster, A. J. 2007. The transcription factor $\operatorname{Con} 7 p$ is a central regulator of infection-related morphogenesis in the rice blast fungus Magnaporthe grisea. Mol. Microbiol. 64:293-307.

Ou, S. H. 1985. Rice Diseases, 2nd ed. Commonwealth Mycological Institute, Surrey, U.K.

Park, D. S., Yu, Y. M., Kim, Y. J., and Maeng, P. J. 2015. Negative regulation of the vacuole-mediated resistance to $\mathrm{K}\left({ }^{+}\right)$stress by a novel $\mathrm{C} 2 \mathrm{H} 2$ zinc finger transcription factor encoded by aslA in Aspergillus nidulans. J. Microbiol. 53:100-110.

Park, G., Xue, C., Zheng, L., Lam, S., and Xu, J. R. 2002. MST12 regulates infectious growth but not appressorium formation in the rice blast fungus Magnaporthe grisea. Mol. Plant-Microbe Interact. 15:183-192.

Park, S. Y., Choi, J., Lim, S. E., Lee, G. W., Park, J., Kim, Y., Kong, S., Kim, S. R., Rho, H. S., Jeon, J., Chi, M. H., Kim, S., Khang, C. H., Kang, S., and Lee, Y. H. 2013. Global expression profiling of transcription factor genes provides new insights into pathogenicity and stress responses in the rice blast fungus. PLoS Pathog. 9:e1003350.

Qi, Z., Wang, Q., Dou, X., Wang, W., Zhao, Q., Lv, R., Zhang, H., Zheng, X., Wang, P., and Zhang, Z. 2012. MoSwi6, an APSES family transcription factor, interacts with MoMps1 and is required for hyphal and conidial morphogenesis, appressorial function and pathogenicity of Magnaporthe oryzae. Mol. Plant Pathol. 13:677-689.

Sambrook, J., Fritsch, E. F., and Maniatis, T. 1989. Molecular Cloning: A Laboratory Manual. Cold Spring Harbor Laboratory Press, Cold Spring Harbor, NY, U.S.A.

Sesma, A., and Osbourn, A. E. 2004. The rice leaf blast pathogen undergoes developmental processes typical of root-infecting fungi. Nature 431: 582-586.

Soanes, D. M., Chakrabarti, A., Paszkiewicz, K. H., Dawe, A. L., and Talbot, N. J. 2012. Genome-wide transcriptional profiling of appressorium development by the rice blast fungus Magnaporthe oryzae. PLoS Pathog. 8:e1002514.

Son, H., Seo, Y. S., Min, K., Park, A. R., Lee, J., Jin, J. M., Lin, Y., Cao, P., Hong, S. Y., Kim, E. K., Lee, S. H., Cho, A., Lee, S., Kim, M. G., Kim, Y., Kim, J. E., Kim, J. C., Choi, G. J., Yun, S. H., Lim, J. Y., Kim, M., Lee, Y. H., Choi, Y. D., and Lee, Y. W. 2011. A phenome-based functional analysis of transcription factors in the cereal head blight fungus, Fusarium graminearum. PLoS Pathog. 7:e1002310.

Talbot, N. J. 2003. On the trail of a cereal killer: Exploring the biology of Magnaporthe grisea. Annu. Rev. Microbiol. 57:177-202.

Talbot, N. J., Ebbole, D. J., and Hamer, J. E. 1993. Identification and characterization of $M P G 1$, a gene involved in pathogenicity from the rice blast fungus Magnaporthe grisea. Plant Cell 5:1575-1590.
Thines, E., Weber, R. W., and Talbot, N. J. 2000. MAP kinase and protein kinase A-dependent mobilization of triacylglycerol and glycogen during appressorium turgor generation by Magnaporthe grisea. Plant Cell 12: 1703-1718.

Tian, C., Li, J., and Glass, N. L. 2011. Exploring the bZIP transcription factor regulatory network in Neurospora crassa. Microbiology 157:747759.

Veneault-Fourrey, C., Barooah, M., Egan, M., Wakley, G., and Talbot, N. J. 2006. Autophagic fungal cell death is necessary for infection by the rice blast fungus. Science 312:580-583.

Wang, Z. Y., Soanes, D. M., Kershaw, M. J., and Talbot, N. J. 2007. Functional analysis of lipid metabolism in Magnaporthe grisea reveals a requirement for peroxisomal fatty acid beta-oxidation during appressoriummediated plant infection. Mol. Plant-Microbe Interact. 20:475-491.

Weber, R. W. S., Pitt, D., and Webster, J. 1998. Teaching techniques for mycology. 3. Amylase secretion by Aspergillus oryzae. Mycologist 12: 8-9.

Weber, R. W. S., Wakley, G. E., and Pitt, D. 1999. Histochemical and ultrastructural characterization of vacuoles and spherosomes as components of the lytic system in hyphae of the fungus Botrytis cinerea. Histochem. J. 31:293-301.

Wei, Y., Zhang, J., Li, K., Huang, L., Li, J., Wang, X., Lin, P., and Wei, Y. 2012. Establishment of a monoclonal antibody against a peptide of the novel zinc finger protein ZNF32 proved to be specific and sensitive for immunological measurements. Med. Sci. Monit. 18:BR167-BR173.

Wilson, R. A., and Talbot, N. J. 2009. Under pressure: Investigating the biology of plant infection by Magnaporthe oryzae. Nat. Rev. Microbiol. 7:185-195.

Xu, J. R., and Hamer, J. E. 1996. MAP kinase and cAMP signaling regulate infection structure formation and pathogenic growth in the rice blast fungus Magnaporthe grisea. Genes Dev. 10:2696-2706.

Xu, J. R., Staiger, C. J., and Hamer, J. E. 1998. Inactivation of the mitogenactivated protein kinase Mps1 from the rice blast fungus prevents penetration of host cells but allows activation of plant defense responses. Proc. Natl. Acad. Sci. U.S.A. 95:12713-12718.

Xu, J. R., Urban, M., Sweigard, J. A., and Hamer, J. E. 1997. The CPKA gene of Magnaporthe grisea is essential for appressorial penetration. Mol. Plant-Microbe Interact. 10:187-194.

Yan, X., Li, Y., Yue, X., Wang, C., Que, Y., Kong, D., Ma, Z., Talbot, N. J., and Wang, Z. 2011. Two novel transcriptional regulators are essential for infection-related morphogenesis and pathogenicity of the rice blast fungus Magnaporthe oryzae. PLoS Pathog. 7:e1002385.

Yu, J. H., Hamari, Z., Han, K. H., Seo, J. A., Reyes-Domínguez, Y., and Scazzocchio, C. 2004. Double-joint PCR: A PCR-based molecular tool for gene manipulations in filamentous fungi. Fungal Genet. Biol. 41:973981.

Zhang, H., Zhao, Q., Liu, K., Zhang, Z., Wang, Y., and Zheng, X. 2009. MgCRZ1, a transcription factor of Magnaporthe grisea, controls growth, development and is involved in full virulence. FEMS Microbiol. Lett. 293:160-169.

Zhao, X., Kim, Y., Park, G., and Xu, J. R. 2005. A mitogen-activated protein kinase cascade regulating infection-related morphogenesis in Magnaporthe grisea. Plant Cell 17:1317-1329.

Zhou, X., Liu, W., Wang, C., Xu, Q., Wang, Y., Ding, S., and Xu, J. R. 2011. A MADS-box transcription factor MoMcm1 is required for male fertility, microconidium production and virulence in Magnaporthe oryzae. Mol. Microbiol. 80:33-53.

Zhou, Z., Li, G., Lin, C., and He, C. 2009. Conidiophore stalk-less 1 encodes a putative zinc-finger protein involved in the early stage of conidiation and mycelial infection in Magnaporthe oryzae. Mol. Plant-Microbe Interact. 22:402-410. 